\title{
Findings of the 2018 Conference on Machine Translation (WMT18)
}

\author{
Ondřej Bojar \\ Charles University \\ Yvette Graham \\ Dublin City University
}

\author{
Christian Federmann \\ Microsoft Cloud + AI
}

Barry Haddow
University of Edinburgh

\author{
Mark Fishel \\ University of Tartu \\ Matthias Huck \\ LMU Munich
}

\author{
Philipp Koehn \\ JHU / University of Edinburgh
}

\author{
Christof Monz \\ University of Amsterdam
}

\begin{abstract}
This paper presents the results of the premier shared task organized alongside the Conference on Machine Translation (WMT) 2018. Participants were asked to build machine translation systems for any of 7 language pairs in both directions, to be evaluated on a test set of news stories. The main metric for this task is human judgment of translation quality. This year, we also opened up the task to additional test sets to probe specific aspects of translation.
\end{abstract}

\section{Introduction}

The Third Conference on Machine Translation (WMT) held at EMNLP $2018^{1}$ host a number of shared tasks on various aspects of machine translation. This conference builds on twelve previous editions of WMT as workshops and conferences (Koehn and Monz, 2006; Callison-Burch et al., 2007, 2008, 2009, 2010, 2011, 2012; Bojar et al., 2013, 2014, 2015, 2016a, 2017).

This year we conducted several official tasks. We report in this paper on the news translation task. Additional shared tasks are described in separate papers in these proceedings:

- biomedical translation (Neves et al., 2018),

- multimodal machine translation (Barrault et al., 2018),

- metrics (Ma et al., 2018),

- quality estimation (Specia et al., 2018),

- automatic post-editing (Chatterjee et al., 2018), and

- parallel corpus filtering (Koehn et al., 2018b).

In the news translation task (Section 2), participants were asked to translate a shared test set, optionally restricting themselves to the provided training data ("constrained" condition). We held
14 translation tasks this year, between English and each of Chinese, Czech, Estonian, German, Finnish, Russian, and Turkish. The EstonianEnglish language pair was new this year. Similarly to Latvian, which we had covered in 2017, Estonian is a lesser resourced data condition on a challenging language pair. System outputs for each task were evaluated both automatically and manually.

This year the news translation task had two additional sub-tracks: multilingual MT and unsupervised MT. Both sub-tracks were included into the general list of news translation submissions and are described in more detail in corresponding subsections of Section 2.

The human evaluation (Section 3) involves asking human judges to score sentences output by anonymized systems. We obtained large numbers of assessments from researchers who contributed evaluations proportional to the number of tasks they entered. In addition, we used Mechanical Turk to collect further evaluations. This year, the official manual evaluation metric is again based on judgments of adequacy on a 100-point scale, a method we explored in the previous years with convincing results in terms of the trade-off between annotation effort and reliable distinctions between systems.

The primary objectives of WMT are to evaluate the state of the art in machine translation, to disseminate common test sets and public training data with published performance numbers, and to refine evaluation and estimation methodologies for machine translation. As before, all of the data, translations, and collected human judgments are publicly available ${ }^{2}$. We hope these datasets serve as a valuable resource for research into datadriven machine translation, automatic evaluation, or prediction of translation quality. News transla-

\footnotetext{
${ }^{2}$ http://statmt.org/wmt 18/results.html
}

\footnotetext{
${ }^{1}$ http://www . statmt . org/wmt18/
} 
tions are also available for interactive visualization and comparison of differences between systems at http://wmt.ufal.cz/ using MT-ComparEval (Sudarikov et al., 2016).

In order to gain further insight into the performance of individual MT systems, we organized a call for dedicated "test suites", each focussing on some particular aspect of translation quality. A brief overview of the test suites is provided in Section 4.

\section{News Translation Task}

The recurring WMT task examines translation between English and other languages in the news domain. As in the previous year, we include Chinese, Czech, German, Finnish, Russian, and Turkish. A new language this year is Estonian.

We created a test set for each language pair by translating newspaper articles and provided training data.

\subsection{Test Data}

The test data for this year's task was selected from online sources, as in previous years. We took about 1500 English sentences and translated them into the other languages, and then additional 1500 sentences from each of the other languages and translated them into English. This gave us test sets of about 3000 sentences for our English-X language pairs, which have been either originally written in English and translated into X, or vice versa. The composition of the test documents is shown in Table 1, the size of the test sets in terms is given in Figure 2.

The stories were translated by professional translators, funded by the EU Horizon 2020 projects CRACKER and QT21 (German, Czech), by Yandex ${ }^{3}$, a Russian search engine company (Turkish, Russian), by BAULT, a research community on building and using language technology funded by the University of Helsinki (Finnish) and the University of Tartu ${ }^{4}$ (Estonian). The Chinese-English task was sponsored by Nanjing University, Xiamen University, the Institutes of Computing Technology and of Automation, Chinese Academy of Science, Northeastern University (China) and Datum Data Co., Ltd. All of the

\footnotetext{
${ }^{3}$ http://www . yandex . com/

${ }^{4}$ Estonian Research Council institutional research grant IUT20-56: "Computational models of the Estonian Language"
}

translations were done directly, and not via an intermediate language.

Since Estonian-English was run for the first time, both the test and development set had to be translated: the size of both was 2000 sentences (4000 in total).

\subsection{Training Data}

As in past years we provided parallel corpora to train translation models, monolingual corpora to train language models, and development sets to tune system parameters. Some training corpora were identical from last year (Europarl, ${ }^{5}$ Common Crawl, SETIMES2, Russian-English parallel data provided by Yandex, Wikipedia Headlines provided by CMU) and some were updated (United Nations, CzEng v1.7 (Bojar et al., 2016b), News Commentary v13, monolingual news data). A new corpus is the EU Press Release parallel corpus for German, Finnish, and Latvian.

For Latvian and Chinese, a number of new corpora were released. For Latvian, this data was prepared by the University of Latvia and Tilde, the Chinese corpora were prepared by the Institutes of Computing Technology and of Automation, Chinese Academy of Science, Northeastern University (China) and Datum Data Co., Ltd.

Some statistics about the training materials are given in Figure 1.

\subsection{Submitted Systems}

We received 103 submissions from 32 institutions. The participating institutions, organized into 35 teams are listed in Table 2 and detailed in the rest of this section. Each system did not necessarily appear in all translation tasks. We also included 39 online MT systems (originating from 5 services), which we anonymized as ONLINE-A,B,F,G.

For presentation of the results, systems are treated as either constrained or unconstrained, depending on whether their models were trained only on the provided data. Since we do not know how they were built, these online and commercial systems are treated as unconstrained during the automatic and human evaluations.

\subsubsection{AALTO (Grönroos et al., 2018)}

Aalto participated in the constrained condition of the multi-lingual subtrack, with a single system trained to translate from English to both Finnish

\footnotetext{
${ }^{5}$ As of Fall 2011, the proceedings of the European Parliament are no longer translated into all official languages.
} 


\section{Europarl Parallel Corpus}

\begin{tabular}{|c|c|c|c|c|c|c|c|c|}
\hline & \multicolumn{2}{|c|}{ German $\leftrightarrow$ English } & \multicolumn{2}{|c|}{ Czech $\leftrightarrow$ English } & \multicolumn{2}{|c|}{ Finnish $\leftrightarrow$ English } & \multicolumn{2}{|c|}{ Estonian $\leftrightarrow$ English } \\
\hline Sentences & 1,92 &, 209 & 646 & 605 & 1,92 & 6,114 & 652 & 944 \\
\hline Words & $50,486,398$ & $53,008,851$ & $14,946,399$ & $17,376,433$ & $37,814,266$ & $52,723,296$ & $13,033,918$ & $17,453,613$ \\
\hline Distinct words & 381,583 & 115,966 & 172,461 & 63,039 & 693,963 & 115,896 & 298,021 & 63,432 \\
\hline
\end{tabular}

News Commentary Parallel Corpus

\begin{tabular}{|c|c|c|c|c|c|c|c|c|}
\hline & \multicolumn{2}{|c|}{ German $\leftrightarrow$ English } & \multicolumn{2}{|c|}{ Czech $\leftrightarrow$ English } & \multicolumn{2}{|c|}{ Russian $\leftrightarrow$ English } & \multicolumn{2}{|c|}{ Chinese $\leftrightarrow$ English } \\
\hline Sentences & 284 & 246 & 218 & 884 & & & &, 777 \\
\hline Words & $7,243,776$ & $7,174,644$ & $4,942,255$ & $5,411,117$ & $6,230,738$ & $6,230,738$ & - & $6,428,459$ \\
\hline Distinct words & 182,059 & 75,590 & 166,173 & 66,054 & 71,021 & 71,021 & - & 70,092 \\
\hline
\end{tabular}

\section{Common Crawl Parallel Corpus}

\begin{tabular}{c|r|r|r|r|r|r} 
& \multicolumn{2}{|c|}{ German $\leftrightarrow$ English } & \multicolumn{2}{c|}{ Czech $\leftrightarrow$ English } & \multicolumn{2}{c}{ Russian $\leftrightarrow$ English } \\
\hline Sentences & \multicolumn{2}{|c|}{$2,399,123$} & \multicolumn{2}{c}{161,838} & \multicolumn{2}{c}{878,386} \\
\hline Words & $54,575,405$ & $58,870,638$ & $3,529,783$ & $3,927,378$ & $21,018,793$ & $21,535,122$ \\
\hline Distinct words & $1,640,835$ & 823,480 & 210,170 & 128,212 & 764,203 & 432,062 \\
\hline
\end{tabular}

ParaCrawl Parallel Corpus

\begin{tabular}{c|r|r|r|r|r|r} 
& \multicolumn{2}{|c|}{ German $\leftrightarrow$ English } & \multicolumn{2}{c|}{ Czech $\leftrightarrow$ English } & \multicolumn{2}{c}{ Estonian $\leftrightarrow$ English } \\
\hline Sentences & \multicolumn{2}{|c|}{$36,351,593$} & \multicolumn{2}{|c|}{$10,020,250$} & \multicolumn{2}{|c}{$1,298,103$} \\
\hline Words & $595,027,749$ & $623,361,284$ & $116,797,931$ & $122,699,058$ & $37,887,435$ & $39,060,095$ \\
\hline Distinct Words & 8065519 & $5,371,211$ & $1,912,633$ & $1,538,696$ & $1,025,961$ & 894,357 \\
\hline
\end{tabular}

\begin{tabular}{c|r|r|r|r} 
& \multicolumn{2}{|c|}{ Finnish $\leftrightarrow$ English } & \multicolumn{2}{c}{ Russian $\leftrightarrow$ English } \\
\hline Sentences & \multicolumn{2}{|c|}{624,058} & \multicolumn{2}{|c}{$1,2061,155$} \\
\hline Words & $8,636,936$ & $11,123,014$ & $182,229,052$ & $210,751,004$ \\
\hline Distinct Words & 379,958 & 127,006 & $3,164,200$ & $2,415,633$ \\
\hline
\end{tabular}

\section{EU Press Release Parallel Corpus}

\begin{tabular}{c|r|r|r|r|r|r} 
& \multicolumn{2}{|c|}{ German $\leftrightarrow$ English } & \multicolumn{2}{c|}{ Finnish $\leftrightarrow$ English } & \multicolumn{2}{c}{ Estonian $\leftrightarrow$ English } \\
\hline Sentences & \multicolumn{2}{|c|}{$1,329,041$} & \multicolumn{2}{c}{583,223} & \multicolumn{2}{c}{226978} \\
\hline Words & $25,048,312$ & $25,777,997$ & $8,052,607$ & $11,244,602$ & $3,940,058$ & 177,723 \\
\hline Distinct words & 398,477 & 168,725 & 315,394 & 94,979 & $5,209,544$ & 57,059 \\
\hline
\end{tabular}

\section{Chinese Parallel Corpora}

\begin{tabular}{c|r|r|r|r|r|r} 
& casia2015 & casict2011 & casict2015 & datum2011 & datum2017 & neu2017 \\
\hline Sentences & $1,050,000$ & $1,936,633$ & $2,036,834$ & $1,000,004$ & 999,985 & $2,000,000$ \\
\hline Words (en) & $20,571,578$ & $34,866,598$ & $22,802,353$ & $24,632,984$ & $25,182,185$ & $29,696,442$ \\
\hline Distinct words (en) & 470,452 & 627,630 & 435,010 & 316,277 & 312,164 & 624,420 \\
\hline
\end{tabular}

\section{Yandex 1M Parallel Corpus}

\begin{tabular}{c|r|r} 
& \multicolumn{2}{|c}{ Russian $\leftrightarrow$ English } \\
\hline Sentences & \multicolumn{2}{|c}{$1,000,000$} \\
\hline Words & $24,121,459$ & $26,107,293$ \\
\hline Distinct & 701,809 & 387,646 \\
\hline
\end{tabular}

\section{CzEng v1.7 Parallel Corpus}

\begin{tabular}{c|c|c} 
& \multicolumn{2}{|c}{ Czech $\leftrightarrow$ English } \\
\hline Sentences & \multicolumn{2}{|c}{$61,243,252$} \\
\hline Words & $737,434,097$ & $3,650,518$ \\
\hline Distinct & $835,192,627$ & $2,580,902$ \\
\hline
\end{tabular}

\section{Wiki Headlines Parallel Corpus}

\begin{tabular}{c|r|r|r|r} 
& \multicolumn{2}{|c|}{ Russian $\leftrightarrow$ English } & \multicolumn{2}{c}{ Finnish $\leftrightarrow$ English } \\
\hline Sentences & \multicolumn{2}{|c|}{514,859} & \multicolumn{2}{c}{153,728} \\
\hline Words & $1,191,474$ & $1,230,644$ & 269,429 & 354,362 \\
\hline Distinct & 282,989 & 251,328 & 127,576 & 96,732 \\
\hline
\end{tabular}

SE Times 2 Parallel Corpus

\begin{tabular}{c|r|r} 
& \multicolumn{2}{|c}{ Turkish $\leftrightarrow$ English } \\
\hline Sentences & \multicolumn{2}{|c}{207,678} \\
\hline Words & $4,626,277$ & $5,147,769$ \\
\hline Distinct & 155,479 & 69,927 \\
\hline
\end{tabular}

\section{United Nations Parallel Corpus}

\begin{tabular}{c|r|r|r|r} 
& \multicolumn{2}{|c|}{ Russian $\leftrightarrow$ English } & \multicolumn{2}{c}{ Chinese $\leftrightarrow$ English } \\
\hline Sentences & \multicolumn{2}{|c|}{$23,239,280$} & \multicolumn{1}{c}{$15,886,041$} \\
\hline Words & $482,966,738$ & $524,719,646$ & - & $372,612,596$ \\
\hline Distinct & $3,857,656$ & $2,737,469$ & - & $1,981,413$ \\
\hline
\end{tabular}

Figure 1: Statistics for the training sets used in the translation task. The number of words and the number of distinct words (case-insensitive) is based on the provided tokenizer. 


\begin{tabular}{|c|c|}
\hline & ources (Number of Documents) \\
\hline English & $\begin{array}{l}\text { ABC News (1), BBC (4), Brisbane Times (1), CBS News (1), Daily Mail (4), Euronews (3), Globe and } \\
\text { Mail (1), Guardian (4), Independent (4), Los Angeles Times (4), MSNBC (3), Novinte (2), New York } \\
\text { Times (2), Reuters (3), Russia Today (2), Scotsman (2), Sydney Morning Herald (2), Telegraph (2), The } \\
\text { Local (2), Time Magazine (2), UPI (1), Washington Post (3) }\end{array}$ \\
\hline Czech & $\begin{array}{l}\text { blesk.cz (16), deník.cz (5), Deník Referendum (1), DNES.cz (7), lidovky.cz (6), Novinky.cz (3), Re- } \\
\text { flex (2), tyden.cz (12), ZDN (2) }\end{array}$ \\
\hline German & $\begin{array}{l}\text { Aachener Nachrichten (1), Abendzeitung Nürnberg (2), Braunschweiger Zeitung (1), Der Standard (1), } \\
\text { Die Presse (1), Euronews (1), Fehmarn24 (1), Handelsblatt (1), Hannoversche Allgemeine (2), Hes- } \\
\text { sische/Niedersächsische Allgemeine (1), In Franken (4), Kreiszeitung (2), Krone (1), Mainpost (1), } \\
\text { Merkur (3), Morgenpost (1), n-tv (1), Neue Westfälische (1), oe24 (2), Peiner Allgemeine (1), Passauer } \\
\text { Neue Presse (2), Rheinzeitung (1), Rundschau (1), Schwarzwälder Bote (16), Segeberger Zeitung (2), } \\
\text { Südkurier (1), Thüringer Allgemeine (1), Thüringer Landeszeitung (1), Volksblatt (2), Volksfreund (3), } \\
\text { Westfälische Nachrichten (1), Westdeutsche Zeitung (8). }\end{array}$ \\
\hline Estonian & Arileht (7), Maaleht (3), Postimees (17), Sloleht (23). \\
\hline Finnish & $\begin{array}{l}\text { Etelä-Saimaa (2), Etelä-Suomen Sanomat (3), Helsingin Sanomat (4), Iltalehti (13), Ilta-Sanomat (29), } \\
\text { Kaleva (12), Kansan Uutiset (1), Karjalainen (13), Kouvolan Sanomat (2). }\end{array}$ \\
\hline Russian & $\begin{array}{l}\text { aif (4), Altapress (1), Argumenti (19), ERR.ee (3), eg-online.ru (2), Euronews (2), Fakty (5), In- } \\
\text { fox (2), Izvestiya (25), Kommersant (16), Lenta (9), lgng (3), MK RU (5), nov-pravda.ru (1), pnp.ru (6), } \\
\text { rg.ru (4), Vedomosti (3), Versia (1), Vesti (3), zr.ru (1) }\end{array}$ \\
\hline & Hürriyet.com (48), Sabah (96), Sözcü (19) \\
\hline
\end{tabular}

Table 1: Composition of the test set. For more details see the XML test files. The docid tag gives the source and the date for each document in the test set, and the origlang tag indicates the original source language.

\section{BigEst Estonian Corpus}

\begin{tabular}{c|r}
\hline Sentences & $40,404,948$ \\
\hline Words & $579,221,489$ \\
\hline Distinct words & $8,134,555$ \\
\hline
\end{tabular}

\section{News Language Model Data}

\begin{tabular}{c|r|r|r|r|r|r|r} 
& English & German & Czech & Russian & Finnish & Turkish & Estonian \\
\hline Sentences & $192,988,741$ & $260,754,881$ & $66,517,569$ & $39,519,008$ & $14,575,981$ & $4,753,928$ & 817,472 \\
\hline Words & $4,428,839,473$ & $4,627,780,738$ & $1,094,215,341$ & $724,582,848$ & $184,523,981$ & $79,067,739$ & $12,880,832$ \\
\hline Distinct words & $6,468,049$ & $20,276,165$ & $4,269,005$ & $3,397,828$ & $4,391,543$ & $1,025,791$ & 653,980 \\
\hline
\end{tabular}

\section{Common Crawl Language Model Data}

\begin{tabular}{c|r|r|r|r|r|r|r|r} 
& English & German & Czech & Russian & Finnish & Estonian & Turkish & Chinese \\
\hline Sent. & $3,074,921,453$ & $2,872,785,485$ & $333,498,145$ & $1,168,529,851$ & $157,264,161$ & $100,779,314$ & $511,196,951$ & $1,672,324,647$ \\
\hline Words & $65,128,419,540$ & $65,154,042,103$ & $6,694,811,063$ & $23,313,060,950$ & $2,935,402,545$ & $2,906,100,138$ & $11,882,126,872$ & \\
\hline Dist. & $342,760,462$ & $339,983,035$ & $50,162,437$ & $101,436,673$ & $47,083,545$ & $27,618,190$ & $88,463,295$ & - \\
\hline
\end{tabular}

Test Set

\begin{tabular}{|c|c|c|c|c|c|c|c|c|}
\hline \multirow{3}{*}{$\begin{array}{c}\text { Sentences. } \\
\text { Words }\end{array}$} & \multicolumn{2}{|c|}{ Czech $\leftrightarrow$ EN } & \multicolumn{2}{|c|}{ German $\leftrightarrow$ EN } & \multicolumn{2}{|c|}{ Finnish $\leftrightarrow$ EN } & \multicolumn{2}{|c|}{ Estonian $\leftrightarrow$ EN } \\
\hline & \multicolumn{2}{|c|}{2983} & \multicolumn{2}{|c|}{2998} & \multicolumn{2}{|c|}{3000} & \multicolumn{2}{|c|}{2000} \\
\hline & 47,229 & 55,920 & 54,933 & 58,628 & 38,149 & 54,790 & 30,531 & 40,158 \\
\hline Distinct words & 18,325 & 12,548 & 15,996 & 13,431 & 17,825 & 12,043 & 14,185 & 10,096 \\
\hline \multirow{2}{*}{\multicolumn{2}{|c|}{ Sentences. }} & \multicolumn{2}{|c|}{ Russian $\leftrightarrow \mathbf{E N}$} & \multicolumn{2}{|c|}{ Turkish $\leftrightarrow$ EN } & \multicolumn{2}{|c|}{ Chinese $\leftrightarrow$ EN } & \\
\hline & & \multicolumn{2}{|c|}{3000} & \multicolumn{2}{|c|}{3000} & \multicolumn{2}{|c|}{3981} & \\
\hline \multicolumn{2}{|c|}{ Words } & 51,988 & 62,925 & 45,944 & 60,232 & - & 98,308 & \\
\hline \multicolumn{2}{|c|}{$\overline{\text { Distinct words }}$} & 21,116 & 13,584 & 19,200 & 13,444 & - & 16,955 & \\
\hline
\end{tabular}

Figure 2: Statistics for the training and test sets used in the translation task. The number of words and the number of distinct words (case-insensitive) is based on the provided tokenizer. 


\begin{tabular}{l|l} 
Team & Institution \\
\hline \hline AALTO & Aalto University (Grönroos et al., 2018) \\
\hline AFRL & Air Force Research Laboratory (Gwinnup et al., 2018) \\
\hline ALIBABA & Alibaba Group (Deng et al., 2018) \\
\hline CUNI-KOCMI & Charles University (Kocmi et al., 2018) \\
\hline CUNI-TRANSFORMER & Charles University (Popel, 2018) \\
\hline FACEBOOK-FAIR $\star$ & Facebook AI Research (Edunov et al., 2018) \\
\hline GTCOM & Global Tone Communication Technology (Bei et al., 2018) \\
\hline HY & University of Helsinki (Raganato et al., 2018) \\
\hline JHU & Johns Hopkins University (Koehn et al., 2018a) \\
\hline JUCBNMT & Jadavpur University (Mahata et al., 2018) \\
\hline KIT & Karlsruhe Institute of Technology (Pham et al., 2018) \\
\hline LI-MUZE & Li Muze (no associated paper) \\
\hline LMU-NMT & LMU Munich (Huck et al., 2018) \\
\hline LMU-UNSUP & LMU Munich (Stojanovski et al., 2018) \\
\hline MICROSOFT-MARIAN & Microsoft (Junczys-Dowmunt, 2018) \\
\hline MLLP-UPV & MLLP, Technical University of Valencia (Iranzo-Sánchez et al., 2018) \\
\hline MMT-PRODUCTION & ModernMT, MMT s.r.l. (no associated paper) \\
\hline NEUROTOLGE.EE & University of Tartu (Tars and Fishel, 2018) \\
\hline NICT & National Institute of Information and Communications Technology \\
(Marie et al., 2018)
\end{tabular}

Table 2: Participants in the shared translation task. Not all teams participated in all language pairs. The translations from the commercial and online systems were not submitted by their respective companies but were obtained by us, and are therefore anonymized in a fashion consistent with previous years of the workshop. " $\star$ " indicates invited participation with a late submission, where the team is not considered a regular participant. 
and Estonian. The system is based on the Transformer (Vaswani et al., 2017) implementation in OpenNMT-py (Klein et al., 2017). It is trained on filtered parallel and filtered back-translated monolingual data. The main contribution is a novel cross-lingual Morfessor (Virpioja et al., 2013) segmentation using cognates extracted from the parallel data. The aim is to improve the consistency of the morphological segmentation. Aalto decode using an ensemble of 3 (et) or 8 (fi) models.

\subsubsection{AFRL (Gwinnup et al., 2018)}

AFRL-SYSCOMB is a system-combination entry consisting of three inputs. The first is an OpenNMT system trained on the provided parallel data except ParaCrawl and the backtranslated corpus used in the AFRL WMT17 system (Gwinnup et al., 2017). This system uses a standard RNN architecture and was fine-tuned with the other available news task test sets. The second is a Marian (Junczys-Dowmunt et al., 2018) system ensembling 5 Univ. Edinburgh "bi-deep" and 6 transformer models all trained on the WMT18 bitexts provided, including ParaCrawl. Some models employed pretrained word embeddings built on BPE'd corpora (Sennrich et al., 2016). A Marian transformer model performed right-to-left rescoring for this system. The third system is trained with Moses (Koehn et al., 2007), using the same data as the Marian system. Hierarchical reordering and Operation Sequence Model were employed. The 5-gram English language model was trained with KenLM (Heafield, 2011) on the same corpus as the AFRL WMT15 system with the same BPE used in the Marian systems. Lastly, RWTH Jane's system combination (Freitag et al., 2014) was applied yielding approximately a +0.5 gain in BLEU.

\subsubsection{ALIBABA (Deng et al., 2018)}

Alibaba systems are based on the Transformer model architecture, with the most recent features from the academic research integrated, such as weighted Transformer, Transformer with relative position attention, etc. The system also employs most techniques that have been proven effective during the past WMT years, such as BPE-based subword, back translation, fine-tuning based on selected data, model ensembling and reranking, at industrial scale. For some morphologically-rich languages, linguistic knowledge is also incorporated into the neural network.

\subsubsection{CUNI-Kocmi (Kocmi et al., 2018)}

The CUNI-Kocmi submission focuses on the low-resource language neural machine translation (NMT). The final submission uses a method of transfer learning: the model is pretrained on a related high-resource language (here Finnish) first, followed by a child low-resource language (Estonian) without any change in hyperparameters. Averaging and backtranslation are also experimented with.

\subsubsection{CUni-Transformer (Popel, 2018)}

CUNI-TRANSFORMER is the Transformer model trained according to Popel and Bojar (2018) plus a novel concat-regime backtranslation with checkpoint averaging, tuned separately for CZ-domain and nonCZ-domain articles, possibly handling also translation-direction ("translationese") issues. For cs $\rightarrow$ en also a coreference preprocessing was used adding the female-gender pronoun where it was pro-dropped in Czech, referring to a human and could not be inferred from a given sentence.

\subsubsection{FACЕВOOK-FAIR $\star($ Edunov et al., 2018)}

FACEBOOK-FAIR is an ensemble of six selfattentional models with back-translation data according to Edunov et al. (2018). Synthetic sources are sampled instead of beam search, oversampling the real bitext at a rate of 16 , i.e., each bitext is sampled 16 times more often per epoch than the back-translated data. At inference time, translations which are copies of the source are filtered out, replacing them with the output of a very small news-commentary only trained model.

The system FACEBOOK-FAIR has been submitted anonymously as ONLINE-Z and approval for disclosing the authors' identity has only been granted after the final results had become available. Due to the non-standard way of submission, the system is not considered a regular participant, but an invited/late submission and marked with " $\star$ " throughout the paper.

\subsubsection{GTCOM (Bei et al., 2018)}

GTCOM-PRIMARY is based on the Transformer "base" model architecture using Marian toolkit, and it also applies some methods that have been proven effective in NMT system, such as BPE, back-translation, right-to-left reranking and ensembling decoding. In this experiment, right-toleft reranking does not help. Another focus is 
given to data filtering through rules, translation model and language model including parallel data and monolingual data. The language model is based the Transformer architecture as well. The final system is trained with four different seeds and mixed data.

\subsubsection{HY (Raganato et al., 2018; Hurskainen and Tiedemann, 2017)}

The University of Helsinki (HY) submitted four systems: HY-AH, HY-NMT, HY-NMT-2STEP and HY-SMT.

HY-AH (Raganato et al., 2018; Hurskainen and Tiedemann, 2017) is a rule-based machine translation system, relying on a rule-based dependency parser for English, a hand-crafted translation lexicon (based on dictionary data extracted from parallel corpora by word alignment), various types of transfer rules, and a morphological generator for Finnish.

HY-NMT (Raganato et al., 2018) submissions are based on the Transformer "base" model, trained with all the parallel data provided by the shared task plus back-translations, with a shared vocabulary between source and target language and a domain label for each source sentence. For the multilingual sub-track synthetic data for English $\rightarrow$ Estonian and Estonian $\rightarrow$ English was also used. Ultimately, a single model for all language pairs was trained and then fine-tuned for each language pair.

HY-NMT-2STEP (Raganato et al., 2018) is a Transformer model trained on interleaved lemmas and morphological tags on the Finnish side. Morphological categories (number, tense etc.) have separate tags, and a tag is only added if the value of the category differs from the default value (in the same way that languages have morphemes only for marked values of morphological categories). The final translation is deterministically generated from the sequence of lemmas and morphological tags which the model outputs.

HY-SMT (Tiedemann et al., 2016) is the Helsinki SMT system submitted at WMT 2016 (the constrained-basic+back-translated version). The system was not retrained and it may thus suffer from poor lexical coverage on recent test data. The main motivation for including this baseline was to have a statistical machine translation
(SMT) submission for the Finnish morphology test suite (Burlot et al., 2018).

\subsubsection{JHU (Koehn et al., 2018a)}

The JHU systems are the result of two relatively independent efforts on German-English language directions and Russian-English, using the Marian and Sockeye (Hieber et al., 2017) neural machine translation toolkits, respectively. The novel contributions are iterative back-translation (for German) and fine-tuning on test sets from prior years (for both languages).

\subsubsection{JUCBNMT (Mahata et al., 2018)}

JUCBNMT is an encoder-decoder sequence-tosequence NMT model with character level encoding. The submission uses preprocessing like tokenization, truecasing and corpus cleaning. Both encoder and decoder use a single LSTM layer each. The batch size was set to 128 , number of epochs was set to 100 , activation function was softmax, optimizer chosen was RMSprop and the loss function used was categorical cross-entropy. Learning rate was set to 0.001 .

\subsubsection{KIT (Pham et al., 2018)}

The KIT submission is the NMT Transformer architecture, enhanced in model depth. Techniques for reducing memory consumption (recalculating intermediate results at layers instead of caching them), 4 times larger model could fit on one GPU and improve the performance by 1.2 BLEU points.

Sentences selection from the new ParaCrawl improved the effectiveness of the corpus by 0.5 BLEU points, with an overall increase of 0.8 BLEU compared to the baseline of not using ParaCrawl.

\subsubsection{LI-MUZE}

LI-MUZE is an ensembles of 4 averaged Transformer models with one right-to-left and one target-to-source averaged Transformer model, the configuration of all the models is the same as the Transformer big-model, trained on the official training data with $4.5 \mathrm{M}$ back-translated data from the monolingual news of 2016 and 2017 data. The English vocabulary size is $36 \mathrm{~K}$ BPE subwords. Chinese is tokenized by Chinese characters and the vocabulary size is $10 \mathrm{~K}$.

\subsubsection{LMU-NMT (Huck et al., 2018)}

For the WMT18 news translation shared task, LMU Munich (Huck et al., 2018) has trained ba- 
sic shallow attentional encoder-decoder systems (Bahdanau et al., 2014) with the Nematus toolkit (Sennrich et al., 2017), like last year (Huck et al., 2017a). LMU has participated with these NMT systems for the English-German language pair in both translation directions. The training data is a concatenation of Europarl, News Commentary, Common Crawl, and some synthetic data in the form of backtranslated monolingual news texts. The 2017 monolingual News Crawl is not employed, nor are the parallel Rapid and ParaCrawl corpora. The German data is preprocessed with a linguistically informed word segmentation technique (Huck et al., 2017b). By using a linguistically more sound word segmentation, advantages over plain BPE segmentation are expected in three important aspects: vocabulary reduction, reduction of data sparsity, and open vocabulary translation. The NMT system can learn linguistic word formation processes from the segmented data. In the English $\rightarrow$ German translation direction, LMU furthermore conducted fine-tuning towards the domain of news articles (Huck et al., 2017a) and reranked the $n$-best list with a right-to-left neural model (Liu et al., 2016) which is trained for reverse word order (Freitag et al., 2013).

\subsubsection{LMU-UNSUP (Stojanovski et al., 2018)}

For the unsupervised track of the WMT18 news translation task, LMU Munich submitted the LMU-UNSUP system (Stojanovski et al., 2018) which is a neural translation model trained without any access to parallel data. The model is trained with $\sim 4 \mathrm{M}$ German and English sentences each, which are sampled from NewsCrawl articles from 2007 to 2017. Bilingual word embeddings trained in an unsupervised manner (Conneau et al., 2017) were used to translate the monolingual data by doing word-by-word translation and this synthetically created parallel data is used in the training as well. The same model is used to do both German $\rightarrow$ English and English $\rightarrow$ German translation. The model is based on (Lample et al., 2018) and it uses denoising and on-the-fly backtranslation. Additionally the model uses the word-byword translated data in the initial training stages to jump-start the training and disables the denoising component as the last training step for further improvements. The NMT embeddings are initialized with embeddings obtained from fast text trained jointly on German and English monolingual BPElevel data.

\subsubsection{MicRosoft-MARIAN (Junczys-Dowmunt, 2018)}

MICROSOFT-MARIAN is the Transformer-big model implemented in Marian with an updated version of Edinburgh's training scheme for WMT2017, following current common practices: truecasing and tokenization using Moses scripts, BPE subwords, backtranslation (using a shallow model), ensembling of four left-to-right deep models and reranking of 12-best list with an ensemble of four right-to-left models.

The novelties are primarily in new data filtering (dual conditional cross-entropy filtering) and sentence weighting methods.

\subsubsection{MLLP-UPV (Iranzo-Sánchez et al., 2018)}

MLLP-UPV is an ensemble of Transformer architecture-based neural machine translation systems. To train the system under "constrained" conditions, the provided parallel data was filtered with a scoring technique using character-based language models, and was augmented based on synthetic source sentences generated from the provided monolingual corpora.

The ensemble consists of 4 independent training runs of the Transformer "base" model, trained with $10 \mathrm{M}$ filtered sentences (including from ParaCrawl) and 20M backtranslated sentences from NewsCrawl2017.

\subsubsection{MMT-PRODUCTION}

MMT-PRODUCTION is the machine translation system offered by MMT s.r.l. (www . modernmt. eu) as of July 2018. It is a Transformer-based neural MT system trained on public and proprietary data, containing about $100 \mathrm{M}$ sentence pairs and about 1.5G English words. It exploits a single model of 'transformer-big' size, and a single pass-decoding; texts are processed using internal tools.

\subsubsection{Neurotolge.ee (Tars and Fishel, 2018)}

NEUROTOLGE.EE is a multi-domain NMT system that treats text domain as language and applies the zero-shot multi-lingual approach to multiple domains in the training corpus. For WMT18, text domains were replaced with unsupervised clustering into 16 clusters using FastText's sentence embeddings. During translation the input segment is 
classified using its sentence embedding and translated as the corresponding cluster/domain.

\subsubsection{NICT (Marie et al., 2018)}

NICT NMT systems were trained with the Transformer architecture using the provided parallel data enlarged with a large quantity of backtranslated monolingual data generated with a new incremental training framework. The primary submissions to the task are the result of a simple combination between NICT SMT and NMT systems.

\subsubsection{NiUTRANS (Wang et al., 2018b)}

NiUTRAns baseline systems are based on the Transformer architecture with the "base" model, equipped with checkpoint averaging and backtranslation techniques. NIUTRANS further improve the translation performance 2.28-3.83 BLEU points from four aspects including model variances (larger inner-hidden-size in FFN, using ReLU and attention dropout, Swish activation function, relative positional representation), diverse ensemble decoding (ensemble decoding with up to 15 models, generated by different strategies), reranking (up to 14 features for reranking), and post-processing (aim at the inconsistent translation of proper nouns, especially the English literals in Chinese sentences).

\subsubsection{NJUNMT}

The NJUNMT-PRIVATE is most likely the system developed by Natural Language Processing Group of Nanjing University based on highlevel API of TensorFlow, https://github. com/zhaocq-nlp/NJUNMT-tf. Further details on training are not available.

\subsubsection{NTT (Morishita et al., 2018)}

NTT combine Transformer "big" model, corpus cleaning technique for provided and synthetic parallel corpora, and right-to-left n-best re-ranking techniques. Through their experiments, NTT found filtering of noisy training sentences and right-to-left re-ranking as the keys to better accuracy.

\subsubsection{PARFDA (Biçici, 2018)}

PARFDA selects a subset of the training and LM data to build task-specific SMT models. PARFDA uses phrase-based Moses and all constrained available resources provided by WMT18. The datasets are available at https://github.com/bicici/ parfdaWMT2018.

\subsubsection{PROMT (Molchanov, 2018)}

PROMT submitted three systems: PROMTHYB-MARIAN, PROMT-HYB-OPENNMT and PROMT-RULE-BASED.

PROMT-HYB-MARIAN is an ensemble of 5 transformer models trained on WMT data and inhouse news data.

PROMT-HYB-OPENNMT is a hybrid system based on PROMT Rule-based engine and a NMT post-editing (PE) engine. The NMT PE component is a sequence-to-sequence model with attention and deep biRNN encoder trained with OpenNMT toolkit.

PROMT-RULE-BASED is a rule-based system, without any specific training or tuning.

\subsubsection{RWTH (Schamper et al., 2018)}

All systems submitted by RWTH Aachen for German to English are based on the Transformer architecture implemented in Sockeye. The final RWTH system has been an ensemble of three Transformer models, where each individual model had been already very strong. The strength of the RWTH systems is probably due to the following four key factors: (a) Using the Transformer architecture. (b) Rather large models and large batch size which was made possible due to synchronous training on 4 GPUs and roughly 8 days of training. (Details: num-embed: 1024; numlayers: 6; attention-heads: 16; transformer-feedforward-num-hidden: 4096; transformer-modelsize: 1024 , no weight-tying. In sum, this results in $291 \mathrm{M}$ trainable parameters.) (c) Careful experiments on data conditions: E.g. oversampling of parallel data, LM driven filtering of ParaCrawl (retained 50\%), testing different amounts of BPE merge operations. (d) Fine-tuning on old testsets (newstest2008-newstest2014).

RWTH English $\rightarrow$ Turkish system is based on 6-layer encoder-decoder Transformer architecture. Since the task has low resources, dropout with the rate of 0.3 to all applicable layers was used. Even though the two languages are not much related, joint BPE and weight tying helped a lot as part of regularization. For the final submission, RWTH used augmented training data with $1 \mathrm{M}$-sentence back-translations and ensembled four models with different random seeds. 


\subsubsection{RWTH-UNSUPER (Graça et al., 2018)}

The RWTH-UNSUPER unsupervised NMT system is built based on recent works by Lample et al. (2018) and Artetxe et al. (2018). RWTHUNSUPER best performing systems follow the batch optimization strategy and are initialized with cross-lingual embeddings. Furthermore, RWTHUNSUPER found that sharing a vocabulary performs better than having separate ones. Freezing embeddings hurts performance and it was found best to initialize embeddings with pre-trained ones and train them as usual.

\subsubsection{TALP-UPC (Casas et al., 2018)}

TALP-UPC is the Transformer "base" model trained with the Tensor2Tensor implementation (Vaswani et al., 2018) and wordpieces vocabulary. The training corpus is multilingual (concatenating Finnish-English and Estonian-English) and includes ParaCrawl with garbage cleaned up via langdetect.

\subsubsection{TENCENT (Wang et al., 2018a)}

TENCENT-ENSEMBLE (called TenTrans) is an improved NMT system on Transformer based on self-attention mechanism. In addition to the basic settings of Transformer training, TENCENTENSEMBLE uses multi-model fusion techniques, multiple features reranking, different segmentation models and joint learning. Additionally, data selection strategies were adopted to fine-tune the trained system, achieving a stable performance improvement.

An additional system paper (Hu et al., 2018) describes a non-primary submission.

\subsubsection{TILDE (Pinnis et al., 2018)}

TILDE submitted four systems: TILDE-C-NMT, TILDE-C-NMT-COMB, TILDE-C-NMT-2BT and TILDE-NC-NMT.

TILDE-C-NMT are constrained English-Estonian and Estonian-English NMT systems that were deployed as ensembles of averaged factored data Transformer models. The models were trained using filtered parallel data and back-translated data in a 1-to-1 proportion. The parallel data were supplemented with synthetic data (generated from the same parallel data) that contain unknown token identifiers in order to acquire models that are more robust to unknown phenomena.
TILDE-C-NMT-COMB is a constrained EstonianEnglish NMT system that is a system combination of multiple constrained factored data NMT systems.

TILDE-C-NMT-2BT systems were trained using Sockeye and Transformer models. Before training the initial systems, parallel data were cleaned using the parallel-corpora-tools. Before back-translation, monolingual data were also filtered. After back-translation, the resulting synthetic corpora were filtered again. Intermediate systems were trained with the first batch of parallel+synthetic data. The back-translation and filtering process was performed a second time with additional monolingual data to train the final systems with parallel and two sets of synthetic data.

TILDE-NC-NMT are unconstrained English $\rightarrow$ Estonian and Estonian $\rightarrow$ English NMT systems that were deployed as averaged Transformer models. These models were also trained using back-translated data similarly to the constrained systems, however, the data, taking into account its relatively large size, was not factored.

\subsubsection{UbiQus}

The UBIQUS-NMT system is probably developed by the Ubiqus company (www.ubiqus. com). No further information is available.

\subsubsection{UCAM (Stahlberg et al., 2018)}

UCAM is a generalization of previous work (de Gispert et al., 2017) to multiple architectures. It is a system combination of two Transformer-like models, a recurrent model, a convolutional model, and a phrase-based SMT system. The output is probably dominated by the Transformer, and to some extend by the SMT system.

\subsubsection{UEDIN (Haddow et al., 2018)}

For Estonian $\leftrightarrow$ English and Finnish $\leftrightarrow$ English, the UEDIN systems are an ensemble of four left-toright systems, reranked with four right-to-left systems, built using Marian. Each ensemble consists of two Transformers and two deep RNNs. The RNNs use the UEDIN multi-head / multi-hop variant. All available parallel data were used, plus back-translated data from 2017 (for into-English) and 2014-2017 (for out-of-English). The natural parallel data was generally over-sampled to give an equal mix of parallel and synthetic data. 
For English $\leftrightarrow$ Estonian, UEDIN selected $30 \%$ of ParaCrawl based on translation model perplexity for a model built on the rest of the data.

The UEDIN systems for other language pairs use an ensemble of four deep RNN left-to-right systems, reranked with 4 deep RNN right-to-left systems. The RNN models use the UEDIN multi-head / multi-hop attention variant. All the provided parallel data (including ParaCrawl) were used, applying langid filtering to remove some incorrect sentence pairs. Synthetic data were also used, created by back-translating the 2017 English news crawl, and the 2017 and 2016 Czech news crawls. For Czech $\rightarrow$ English, the synthetic data was oversampled $2 \mathrm{x}$.

\subsubsection{UMD (Xu and Carpuat, 2018)}

The UMD best system is an ensemble of three 6-layer left-to-right Transformer models reranked with target-to-source and left-to-right models. Each Transformer model is trained with a 2:1 mixture of parallel and backtranslated monolingual data. For parallel data, duplicates are removed and "bad" sentence pairs filtered out. Monolingual data is sub-sampled from news 2017 (English) and news 2011 (Chinese). Subwords (BPE) are used for both English and Chinese sentences.

\subsubsection{UNISOUND}

The UNISOUND systems are probably developed by the Unisound company (www . uni sound . com). No further information is available.

\subsubsection{Unsuptartu (Del et al., 2018)}

UNSUPTARTU is an unsupervised MT system using $n$-gram embedding cross-lingual mapping to create a phrase table. An RNN LM is used in decoding.

\subsection{Multilingual Sub-track}

This year the news translation track included an explicit sub-track on multilingual translation. This covered any submissions that used any data (monolingual or parallel) from a third language to help the language pair in question: for example, using English-Finnish data to improve EnglishEstonian translation. All entries to this sub-track had to use only the WMT-provided data sets, and thus had to be constrained. Submissions to this sub-track are joined with the main translation track and evaluated without separation in the same way.

While there was no restriction in terms of language pairs, three language pairs were "verbally endorsed": English to/from Turkish, Estonian and German. The motivation behind the choice of languages was to test the effect of multilingual (and unsupervised) methods on low-resource language pairs (Turkish-English, Estonian-English) and to contrast the results with a resource-rich pair (German-English).

\subsection{Unsupervised Sub-track}

In the unsupervised MT sub-track the participants were constrained to using only the monolingual training data from WMT; this additionally excluded the monolingual corpora that are largely parallel (monolingual parts of Europarl and News Commentary). The aim of this task was to see how far can one get in terms of translation quality without any parallel data used for training. As an exception it was allowed to use a parallel dev set for parameter tuning and/or model selection. The language pairs of this sub-track coincided with the multilingual sub-track: English to/from Turkish, Estonian and German.

\section{Human Evaluation}

A human evaluation campaign is run each year to assess translation quality and to determine the final ranking of systems taking part in the competition. This section describes how preparation of evaluation data, collection of human assessments, and computation of the official results of the shared task was carried out this year.

Work on evaluation over the past few years has provided fresh insight into ways to collect direct assessments (DA) of machine translation quality (Graham et al., 2013, 2014, 2016), and two years ago the evaluation campaign included parallel assessment of a subset of News task language pairs evaluated with relative ranking (RR) and DA. DA has some clear advantages over RR, namely the evaluation of absolute translation quality and the ability to carry out evaluations through quality controlled crowd-sourcing. As established in 2016 (Bojar et al., 2016a), DA results (via crowd-sourcing) and RR results (produced by researchers) correlate strongly, with Pearson correlation ranging from 0.920 to 0.997 across several source languages into English and at 0.975 for English-to-Russian (the only pair evaluated outof-English). Last year, we thus employed DA for evaluation of systems taking part in the news task and do so again this year. Where possible, 


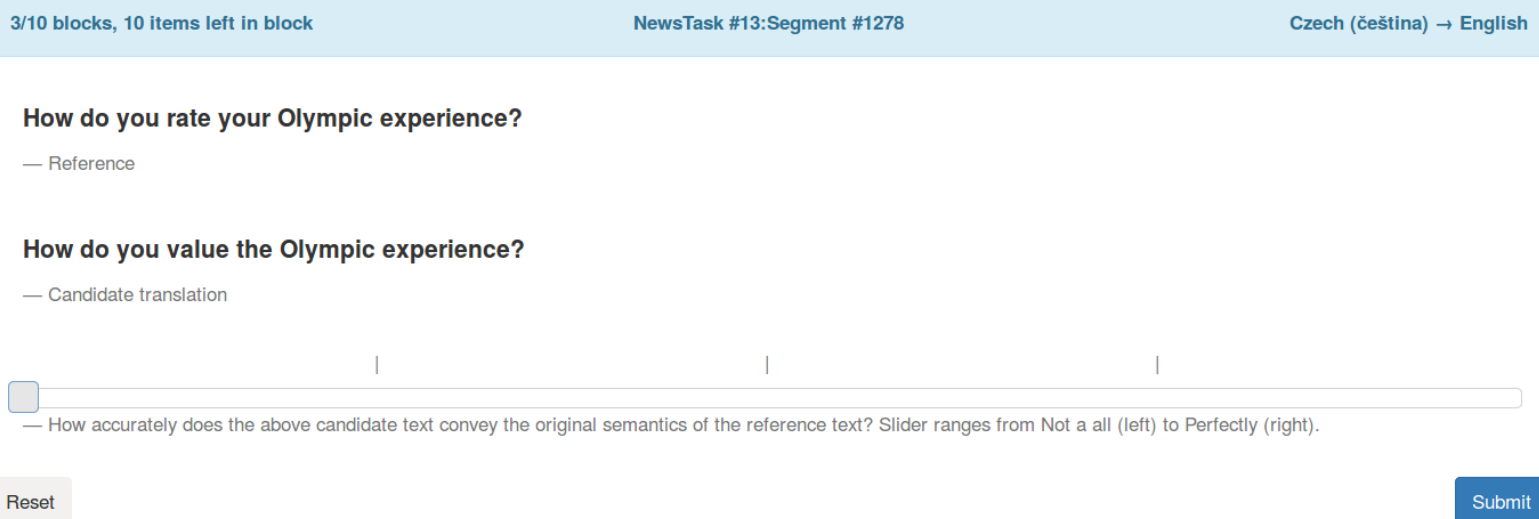

Figure 3: Screen shot of Direct Assessment in the Appraise interface used in the human evaluation campaign. The annotator is presented with a reference translation and a single system output randomly selected from competing systems (anonymized), and is asked to rate the translation on a sliding scale.

This HIT consists of 100 English assessments. You have completed 0.

Read the text below. How much do you agree with the following statement:

The black text adequately expresses the meaning of the gray text in English.

To snobs like me who declare that they'd rather play sports than watch them, it's hard to see the appeal of watching games rather than taking up a controller myself.

Snob like me, who say that it is better to be in sports than watching him, it is hard to understand the appeal of having to watch the game, rather than to take a joystick in hand.

Figure 4: Screen shot of Direct Assessment as carried out by workers on Mechanical Turk.

we collect DA judgments via the crowd-sourcing platform, Amazon's Mechanical Turk, and as in previous year's we ask participating teams to provide manual evaluation of system outputs via Appraise. Researcher involvement was needed particularly for translations into Czech, German, Estonian, Finnish and Turkish.

Human assessors are asked to rate a given translation by how adequately it expresses the meaning of the corresponding reference translation (i.e. no bilingual speakers are needed) on an analogue scale, which corresponds to an underlying absolute $0-100$ rating scale. Since DA involves evaluation of a single translation per screen, this allows the sentence length restriction usually applied during manual evaluation to be removed for both researchers and crowd-sourced workers. ${ }^{6}$ Figure 3

\footnotetext{
${ }^{6}$ The maximum sentence length with RR was 30 in
}

shows one DA screen as completed by researchers on Appraise, while Figure 4 provides a screenshot of DA shown to crowd-sourced workers on Amazon's Mechanical Turk.

The annotation is organized into "HITs" (following the Mechanical Turk's term "human intelligence task"), each containing 100 such screens and requiring about half an hour to finish. Appraise users were allowed to pause their annotation at any time, Amazon interface did not allow any pauses. More details of composition of HITs are given in Section 3.3 below.

\subsection{Evaluation Campaign Overview}

In terms of the News translation task manual evaluation, a total of 584 individual researcher ac-

WMT16. 
counts were involved, and 915 turker accounts. $^{7}$ Researchers in the manual evaluation came from 33 different research groups and contributed judgments of 118,705 translations, while 225,900 translation assessment scores were submitted in total by the crowd. ${ }^{8}$

Under ordinary circumstances, each assessed translation would correspond to a single individual scored segment. However, since distinct systems can produce the same output for a particular input sentence, we are often able to take advantage of this and use a single assessment for multiple systems. Similar to last year's evaluation, we only combine human assessments in this way if the string of text belonging to multiple systems is exactly identical. For example, even small differences in punctuation disqualify combination of similar system outputs, and this is due to a general lack of evidence about what kinds of minor differences may or may not impact human evaluation.

Table 3 shows the numbers of segments for which distinct MT systems participating in the News Translation Task produced identical outputs. The biggest saving in terms of exact duplicate translations, being produced by multiple systems, was for German to English, where a 17.4\% saving of resources by combining identical outputs before human evaluation.

\subsection{Data Collection}

System rankings are produced from a large set of human assessments of translations, each of which indicates the absolute quality of the output of a system. Annotations are collected in an evaluation campaign that enlists the help of participants in the shared task. Each team is asked to contribute 8 hours annotation time, which we estimated at 16 100-translation HITs per primary system submitted. We continue to use the open-source Appraise ${ }^{9}$ (Federmann, 2012) tool for our data collection, in addition to Amazon Mechanical Turk. ${ }^{10}$ Table 4 shows total numbers of human assessments col-

\footnotetext{
${ }^{7}$ Numbers do not include the 1,533 workers on Mechanical Turk and 7 Appraise evaluators who did not pass quality control.

${ }^{8}$ Numbers include quality control items for workers who passed quality control but omit the additional 347,700 assessments collected on Mechanical Turk where a worker did not pass quality control and equivalent 1,466 judgments for the small number of Appraise workers who did not meet the quality control threshold. A $40 \%$ pass rate for quality control is typical of DA evaluations on Mechanical Turk.

${ }^{9}$ https://github.com/cfedermann/Appraise

${ }^{10}$ https: //WWW . mturk. com
}

lected in WMT18 contributing to final scores for systems. ${ }^{11}$

The effort that goes into the manual evaluation campaign each year is impressive, and we are grateful to all participating individuals and teams. We believe that human annotation provides the best decision basis for evaluation of machine translation output and it is great to see continued contributions on this large scale.

\subsection{Crowd Quality Control}

This year, two distinct HIT structures were run in the overall evaluation campaign, the standard DA set-up was employed for Mechanical Turk and a portion of the Appraise evaluation, while an additional HIT structure was used for the remaining part of the Appraise evaluation. Below we firstly describe the standard DA HIT structure and quality control mechanism before describing the additional version used for part of the Appraise evaluation. In both set-ups, translations are arranged in sets of 100-translation HITs to provide control over assignment and positioning of quality control items to human annotators.

In the standard DA HIT structure, three kinds of quality control translation pairs are employed as described in Table 5: we repeat pairs (expecting a similar judgment), damage MT outputs (expecting significantly worse scores) and use references instead of MT outputs (expecting high scores).

In total, 60 items in a 100-translation HIT serve in quality control checks but 40 of those are regular judgments of MT system outputs (we exclude assessments of bad references and ordinary reference translations when calculating final scores). The effort wasted for the sake of quality control is thus $20 \%$.

Also in the standard DA HIT structure, within each 100-translation HIT, the same proportion of translations are included from each participating system for that language pair. This ensures the final dataset for a given language pair contains roughly equivalent numbers of assessments for each participating system. This serves three purposes for making the evaluation fair. Firstly, for the point estimates used to rank systems to be reliable, a sufficient sample size is needed and the

\footnotetext{
${ }^{11}$ Appraise ran evaluation of $150-1=149$ systems due to a single tr-en system having been omitted in the initial human evaluation run. The 95 crowd-sourced systems includes all into-English language pair (including the tr-en missing system), en-ru and en-zh systems.
} 


\begin{tabular}{ccccccc}
\hline Language Pair & Systems & Segments & $\begin{array}{c}\text { Total } \\
\text { Segments }\end{array}$ & $\begin{array}{c}\text { Distinct } \\
\text { Segments }\end{array}$ & $\begin{array}{c}\text { Saving }(\%) \\
\text { WMT18 }\end{array}$ & $\begin{array}{c}\text { Saving (\%) } \\
\text { WMT17 }\end{array}$ \\
\hline Chinese $\rightarrow$ English & 14 & 3,981 & 55,734 & 49,767 & $\mathbf{1 0 . 7}$ & 3.9 \\
Czech $\rightarrow$ English & 5 & 2,983 & 14,915 & 13,987 & $\mathbf{6 . 2}$ & 4.3 \\
German $\rightarrow$ English & 16 & 2,998 & 47,968 & 39,627 & $\mathbf{1 7 . 4}$ & 10.7 \\
Estonian $\rightarrow$ English & 14 & 2,000 & 28,000 & 25,612 & $\mathbf{8 . 5}$ & - \\
Finnish $\rightarrow$ English & 9 & 3,000 & 27,000 & 25,233 & $\mathbf{6 . 5}$ & 1.4 \\
Russian $\rightarrow$ English & 8 & 3,000 & 24,000 & 21,966 & $\mathbf{8 . 5}$ & 5.8 \\
Turkish $\rightarrow$ English & 6 & 3,000 & 18,000 & 17,000 & $\mathbf{5 . 6}$ & 4.6 \\
English $\rightarrow$ Chinese & 14 & 3,981 & 55,734 & 48,022 & $\mathbf{1 3 . 8}$ & 1.7 \\
English $\rightarrow$ Czech & 5 & 2,983 & 14,915 & 13,982 & $\mathbf{6 . 3}$ & 10.2 \\
English $\rightarrow$ German & 16 & 2,998 & 47,968 & 39,963 & $\mathbf{1 6 . 7}$ & 12.8 \\
English $\rightarrow$ Estonian & 14 & 2,000 & 28,000 & 25,837 & $\mathbf{7 . 7}$ & - \\
English $\rightarrow$ Finnish & 12 & 3,000 & 36,000 & 32,749 & $\mathbf{9 . 0}$ & 3.7 \\
English $\rightarrow$ Russian & 9 & 3,000 & 27,000 & 24,594 & $\mathbf{8 . 9}$ & 4.5 \\
English $\rightarrow$ Turkish & 8 & 3,000 & 24,000 & 21,880 & $\mathbf{8 . 8}$ & 2.1 \\
\hline
\end{tabular}

Table 3: Total segments prior to sampling for manual evaluation and savings made by combining outputs produced by different systems that were identical.

most efficient way to reach a sufficient sample size for all systems is to keep total numbers of judgments roughly equal as more and more judgments are collected. Secondly, it helps to make the evaluation fair because each system will suffer or benefit equally from an overly lenient/harsh human judge. Thirdly, despite DA judgments being absolute, it is known that judges "calibrate" the way they use the scale depending on the general observed translation quality. With each HIT including all participating systems, this effect is averaged out. Furthermore apart from quality control items, HITs are constructed using translations sampled from the entire set of outputs for a given language pair.

The alternate DA HIT structure employed by Appraise this year for a subset of researcher HITs is shown in Table 6. This set-up reduces the number of quality control items in a HIT and is therefore more efficient ( $12 \%$ overhead) by omitting repeat pairs and good reference pairs. This comes at the cost of a reduced ability to analyze the quality of data provided by human annotators. In addition for this set-up, an additional constraint (not originally applied in standard DA) was imposed. As much as possible within a 100-translation HIT the HIT included the output of all participating systems for each source input. This constraint has the advantage of producing assessments from the same human assessor for translations of the same source input but is not ideal in terms of the original aim of DA - to as much as possible produce absolute scores for translations (as opposed to relative ones) - because it positions assessment of competing translations in close proximity within a HIT and judges may attempt to remember their judgment for a different candidate translation of a given input sentence.

In all set-ups employed in the evaluation campaign, and as in previous years, bad reference pairs were created automatically by replacing a phrase within a given translation with a phrase of the same length randomly selected from n-grams extracted from the full test set of reference translations belonging to that language pair. This means that the replacement phrase will itself comprise a fluent sequence of words (making it difficult to tell that the sentence is low quality without reading the entire sentence) while at the same time making its presence highly likely to sufficiently change the meaning of the MT output so that it causes a noticeable degradation. The length of the phrase to be replaced is determined by the number of words in the original translation, as follows: 


\begin{tabular}{lrrrrr}
\hline Language Pair & Systems & Comps & Comps/Sys & Assessments & Assess/Sys \\
\hline Chinese $\rightarrow$ English & 14 & - & - & 32,919 & $2,351.4$ \\
Czech $\rightarrow$ English & 5 & - & - & 12,209 & $2,441.8$ \\
German $\rightarrow$ English & 16 & - & - & 48,469 & $3,029.3$ \\
Estonian $\rightarrow$ English & 14 & - & - & 28,868 & $2,062.0$ \\
Finnish $\rightarrow$ English & 9 & - & - & 18,868 & $2,096.4$ \\
Russian $\rightarrow$ English & 8 & - & - & 17,711 & $2,213.9$ \\
Turkish $\rightarrow$ English & 6 & - & - & 29,784 & $4,964.0$ \\
& & & & & \\
English $\rightarrow$ Chinese & 14 & - & - & 32,411 & $2,315.1$ \\
English $\rightarrow$ Czech & 5 & - & - & 10,080 & $2,016.0$ \\
English $\rightarrow$ German & 16 & - & - & 13,754 & 859.6 \\
English $\rightarrow$ Estonian & 14 & - & - & 15,800 & $1,128.6$ \\
English $\rightarrow$ Finnish & 12 & - & - & 9,995 & 832.9 \\
English $\rightarrow$ Russian & 9 & - & - & 27,977 & $3,108.6$ \\
English $\rightarrow$ Turkish & 8 & - & - & 3,644 & 455.5 \\
& & & & & \\
Total Researcher & 149 & - & - & 101,189 & 679.1 \\
Total Crowd & 95 & - & - & 201,300 & $2,118.9$ \\
Total WMT18 & $\mathbf{1 5 0}$ & - & - & $\mathbf{3 0 2 , 4 8 9}$ & $\mathbf{2 , 0 1 6 . 6}$ \\
& & & & & \\
WMT17 & 153 & - & - & 307,707 & $2,011.2$ \\
WMT16 & 138 & 569,287 & $4,125.2$ & 284,644 & $2,062.6$ \\
WMT15 & 131 & 542,732 & $4,143.0$ & 271,366 & $2,071.5$ \\
WMT14 & 110 & 328,830 & $2,989.3$ & 164,415 & $1,494.7$ \\
WMT13 & 148 & 942,840 & $6,370.5$ & 471,420 & $3,185.3$ \\
WMT12 & 103 & 101,969 & 999.6 & 50,985 & 495.0 \\
WMT11 & 133 & 63,045 & 474.0 & 31,522 & 237.0 \\
\hline
\end{tabular}

Table 4: Amount of data collected in the WMT18 manual evaluation campaign (assessments after removal of quality control items and "de-collapsing" multi-system outputs). The final seven rows report summary information from previous years of the workshop.

\begin{tabular}{cc}
\hline $\begin{array}{c}\text { Translation } \\
\text { Length }(\mathrm{N})\end{array}$ & $\begin{array}{c}\text { \# Words Replaced } \\
\text { in Translation }\end{array}$ \\
\hline 1 & 1 \\
$2-5$ & 2 \\
$6-8$ & 3 \\
$9-15$ & 4 \\
$16-20$ & 5 \\
$>20$ & $\lfloor\mathrm{~N} / 4\rfloor$ \\
\hline
\end{tabular}

\subsection{Annotator Agreement}

When an analogue scale (or 0-100 point scale, in practice) is employed, agreement cannot be measured using the conventional Kappa coefficient, ordinarily applied to human assessment when judgments are discrete categories or preferences. Instead, to measure consistency we filter crowd-sourced human assessors by how consistently they rate translations of known distinct quality using the bad reference pairs described previously. Quality filtering via bad reference pairs is especially important for the crowd-sourced portion of the manual evaluation. Due to the anonymous nature of crowd-sourcing, when collecting assessments of translations, it is likely to encounter workers who attempt to game the service, as well as submission of inconsistent evaluations and even robotic ones. We therefore employ DA's quality control mechanism to filter out low quality data, facilitated by the use of DA's analogue rating scale.

Assessments belonging to a given crowdsourced worker who has not demonstrated that he/she can reliably score bad reference translations significantly lower than corresponding genuine system output translations are filtered out. A paired significance test is applied to test if degraded translations are consistently scored lower 


\begin{tabular}{lll}
\hline Repeat Pairs: & Original System output (10) & An exact repeat of it (10); \\
Bad Reference Pairs: & Original System output (10) & A degraded version of it (10); \\
Good Reference Pairs: & Original System output (10) & Its corresponding reference translation (10). \\
\hline
\end{tabular}

Table 5: Standard DA HIT structure quality control translation pairs hidden within 100-translation HITs, numbers of items are provided in parentheses.

\begin{tabular}{lll}
\hline Bad Reference Pairs: & Original System output (12) & A degraded version of it (12). \\
\hline
\end{tabular}

Table 6: Additional DA HIT structure used for a portion of researchers in Appraise data collection, where quality control translation pairs hidden within 100-translation HITs, numbers of items are provided in parentheses in adapted version of DA used for a subset of researchers HITs.

than their original counterparts and the p-value produced by this test is used as an estimate of human assessor reliability. Assessments of workers whose p-value does not fall below the conventional 0.05 threshold are omitted from the evaluation of systems, since they do not reliably score degraded translations lower than corresponding MT output translations.

This year's assessment includes the first largescale DA evaluation where quality control items were applied to assessments of a known-reliable group, comprised of the portion of researchers who completed HITs on Appraise with the original DA HIT structure. Although this group should be considered highly reliable compared to Mechanical Turk for example, we must however keep in mind that a small part of this group are in fact hired to complete assessments and their reliability could vary more than what would be expected of volunteer researchers.

Table 7 shows the number of workers in the crowd-sourced and researcher groups who met our filtering requirement by showing a significantly lower score for bad reference items compared to corresponding MT outputs, and the proportion of those who simultaneously showed no significant difference in scores they gave to pairs of identical translations.

A main observation to be taken from Table 7 is the difference in proportions of human assessors on Mechanical Turk versus researchers who passed the quality filtering criteria for DA, by scoring degraded translations significantly lower than the original MT output counterparts, as 37\% of Mechanical Turk workers were deemed reliable compared to $93 \%$ of evaluators in the researcher group. This low rate of workers passing quality filtering is inline with past DA evaluations, and the high proportion of annotators passing quality control is expected of a mostly known- reliable group. For crowd-sourced workers, consistent with past DA evaluations, Table 7 shows a substantially higher number of low quality workers encountered for evaluation of languages other than English on Mechanical Turk. For example, in the case of Russian and Chinese only a respective $22 \%$ and $10 \%$ of workers were considered reliable enough to include their assessments in the evaluation, compared to around $42 \%$ on average for English evaluations.

When we examine repeat assessments of the same translation, both filtered groups show similar levels of reliability with $96 \%$ of filtered Mechanical Turk workers and $95 \%$ of researchers showing no significant difference in scores for repeat assessment of the same translation. The idea is that the repeated input should receive a very similar score. Assuming that annotators do not remember their previous assessment for the repeated sentence, the "Exact Rep." corresponds to intra-annotator agreement and it reaches very high scores. $^{12}$

Within the researcher group, although assessors have high levels of reliability overall, reliability in this respect varies quite a bit for different languages. For example, only $75 \%$ of assessors in the researcher group completing assessments for Estonian showed no significant difference for repeat assessment of the same translation, and $87 \%$ for Turkish, both lower levels of reliability than usually encountered on Mechanical Turk even though the research group is expected to be more reliable than crowd-sourced workers. However, on closer inspection, the number of human assessors who took part in the Turkish and Estonian evaluations is small and the seemingly large difference in percentages in fact correspond to as few as three indi-

\footnotetext{
${ }^{12}$ Repeat items are separated by a minimum of 40 intervening assessments to reduce the likelihood of annotators simply remembering previous scores for repeat assessment of translations.
} 
(A)

Sig. Diff.

All Bad Ref.

Czech $\rightarrow$ English
German $\rightarrow$ English
Estonian $\rightarrow$ English
Finnish $\rightarrow$ English
Russian $\rightarrow$ English
Turkish $\rightarrow$ English
Chinese $\rightarrow$ English
English $\rightarrow$ Russian
English $\rightarrow$ Chinese

\section{Crowd}

\section{2,477}

$915(37 \%)$

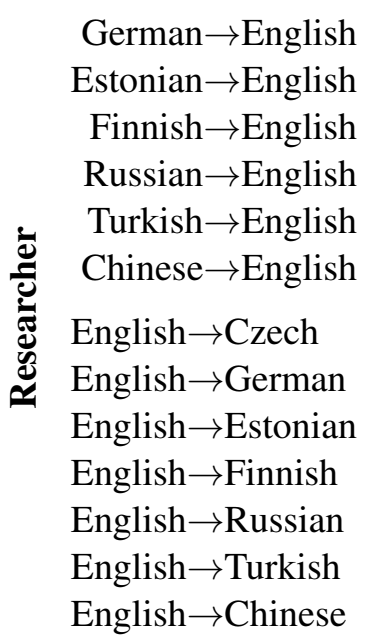

41
16
3
8
7
4
17
48
6
29
26
17
34

$39(95 \%)$

$13(81 \%)$

$3(100 \%)$

$8(100 \%)$

$7(100 \%)$

$3(75 \%)$

$17(100 \%)$

47 (98\%)

$4(67 \%)$

$27(93 \%)$

(B)

(A) \& No Sig. Diff.

Exact Rep.

$70(95 \%)$
$216(95 \%)$
$150(96 \%)$
$99(97 \%)$
$93(97 \%)$
$166(97 \%)$
$148(97 \%)$
$45(96 \%)$
$37(95 \%)$

\section{0 ( $96 \%)$}

$37(95 \%)$

$13(100 \%)$

$3(100 \%)$

$8(100 \%)$

$7(100 \%)$

$3(100 \%)$

$17(100 \%)$

$44(94 \%)$

$3(75 \%)$

$25(93 \%)$

$24(96 \%)$

$13(87 \%)$

$30(97 \%)$

$256 \quad 239(93 \%)$

227 ( $95 \%)$

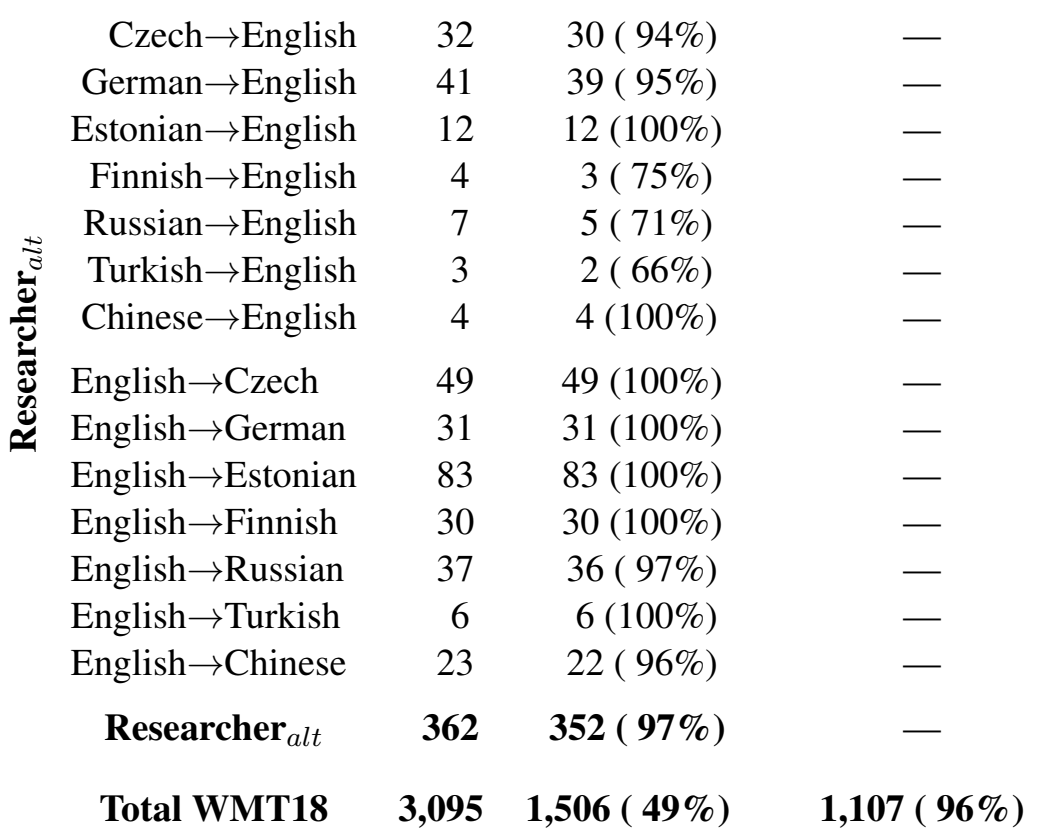

Table 7: Number of unique workers, (A) those whose scores for bad reference items were significantly lower than corresponding MT outputs; (B) those of (A) whose scores also showed no significant difference for exact repeats of the same translation. Researcher denotes the portion of the evaluation carried out with the standard DA HIT structure, while Researcher alt denotes the remaining part that employed the altered HIT structure in which some quality control items are omitted. 
viduals.

\subsection{Producing the Human Ranking}

All research and crowd data that passed quality control were combined to produce the overall shared task results. In order to iron out differences in scoring strategies of distinct human assessors, human assessment scores for translations were first standardized according to each individual human assessor's overall mean and standard deviation score, for both researchers and crowd. Average standardized scores for individual segments belonging to a given system are then computed, before the final overall DA score for that system is computed as the average of its segment scores (Ave $z$ in Table 8). Results are also reported for average scores for systems, computed in the same way but without any score standardization applied (Ave \% in Table 8).

Table 8 includes final DA scores for all systems participating in WMT18 News Translation Task. Clusters are identified by grouping systems together according to which systems significantly outperform all others in lower ranking clusters, according to Wilcoxon rank-sum test.

Note that for English $\rightarrow$ German, the system FACEBOOK-FAIR is not considered a regular participant, but an invited/late submission, see Section 2.3.6.

Appendix A shows the underlying head-to-head significance test results for all pairs of systems.

\subsection{Source-based Direct Assessment}

A secondary bilingual manual evaluation was carried out involving an adaptation of the standard monolingual DA evaluation in which the source language input segment was used in place of the reference. Figure 5 provides a screenshot of this evaluation as implemented in Appraise, which we refer to as source-based DA. In this set-up system outputs are evaluated by bilinguals who have access to the source language input segment only and no reference translation. The main motivation for doing so was to free up reference translations to allow them to be used instead as a "human system" in the evaluation. By structuring the evaluation as a bilingual task it allows a human system to be manually evaluated under exactly the same conditions as all other systems thus providing an estimate of human performance. ${ }^{13}$

\footnotetext{
${ }^{13}$ An alternate method is to keep DA monolingual but to employ secondary reference translations. No secondary ref-
}

The aim of source-based DA is to produce accurate rankings for systems as well as the human system to allow direct comparison of system and human performance, motivated by recent indications that Machine Translation quality may in some cases be approaching human performance (Wu et al., 2016; Hassan et al., 2018). For sourcebased DA, annotators will ideally be bilingual, i.e. understand the source language sufficiently well, in addition to being native speakers of the target language. However, we did not specifically stipulate in this year's evaluation that human annotators be native speakers of the target language.

We run source-based DA for evaluation of English to Czech translation. This language pair was selected because sufficient annotators were available, helped by the fact that the set of systems participating in this language pair is small. This part of the campaign employs the alternate HIT structure described in Section 3.3 with reduced quality control items, i.e. it does not include exact repeats of translations or reference translations for quality control purposes.

A total of 17 annotators worked on the sourcebased DA pilot. $100 \%$ of annotators proved reliable, meaning that they scored bad reference items significantly lower than corresponding MT outputs (see Table 7 part (A) for corresponding reference-based DA percentages). For six candidate systems we collected 2, 574 assessments, resulting in an average of 429 annotations per individual system. Enforcing segment overlap during HIT creation resulted in 423 segments for which all six candidate translations have been scored. In total, annotators worked on 438 distinct segments.

Table 9 provides source-based DA scores for all primary English $\rightarrow$ Czech systems participating in WMT18 News Translation Task as well as the human system comprised of reference translations. Clusters are identified by grouping systems together according to which systems significantly outperform all others in lower ranking clusters, according to Wilcoxon rank-sum test.

As can be seen from clusters in Table 9, one system, CUNI-TRANSFORMER, appears to achieve quality better than that of the human reference, NEWSTEST2018-REF, while another, UEDIN, appears to be on par with human performance, and although both systems certainly achieve very impressive results, claims of human parity should be

erence translations were available for the test set, however. 
Chinese $\rightarrow$ English

\begin{tabular}{ccrl} 
& \multicolumn{3}{c}{ Chinese $\rightarrow$ English } \\
& Ave. $\%$ & Ave. $\mathrm{z}$ & System \\
\hline 1 & 78.8 & 0.140 & NIUTRANS \\
& 77.7 & 0.111 & ONLINE-B \\
& 77.9 & 0.109 & UCAM \\
& 78.0 & 0.108 & UNISOUND-A \\
& 77.5 & 0.099 & TENCENT-ENSEMBLE \\
& 77.5 & 0.094 & UNISOUND-B \\
& 77.9 & 0.091 & LI-MUZE \\
& 77.0 & 0.089 & NICT \\
& 76.7 & 0.078 & UMD \\
\hline 10 & 75.0 & -0.005 & ONLINE-Y \\
& 74.5 & -0.017 & UEDIN \\
\hline 12 & 73.6 & -0.061 & ONLINE-A \\
\hline 13 & 65.9 & -0.327 & ONLINE-G \\
\hline 14 & 64.4 & -0.377 & ONLINE-F \\
\hline
\end{tabular}

English $\rightarrow$ Chinese

\begin{tabular}{rcrl} 
& Ave. $\%$ & Ave. $\mathrm{z}$ & System \\
\hline 1 & 80.7 & 0.219 & TENCENT-ENSEMBLE \\
& 80.3 & 0.206 & UNISOUND \\
& 80.5 & 0.199 & GTCOM-PRIMARY \\
& 79.7 & 0.185 & ALIBABA-ENS-RERANK \\
& 79.2 & 0.173 & ALIBABA-GENERAL-A \\
& 79.5 & 0.166 & ONLINE-B \\
& 79.0 & 0.165 & ALIBABA-GENERAL-B \\
\hline 8 & 78.1 & 0.094 & UMD \\
& 77.5 & 0.082 & NICT \\
& 77.1 & 0.069 & ONLINE-Y \\
& 75.5 & 0.037 & ONLINE-A \\
\hline 12 & 70.7 & -0.202 & UEDIN \\
\hline 13 & 63.3 & -0.419 & ONLINE-F \\
& 63.4 & -0.435 & ONLINE-G \\
\hline
\end{tabular}

Czech $\rightarrow$ English

\begin{tabular}{rcrl} 
& Ave. $\%$ & \multicolumn{1}{c}{ Ave. $\mathrm{z}$} & System \\
\hline 1 & 71.8 & 0.298 & CUNI-TRANSFORMER \\
\hline 2 & 67.9 & 0.165 & UEDIN \\
\hline 3 & 66.6 & 0.115 & ONLINE-B \\
\hline 4 & 62.1 & -0.023 & ONLINE-A \\
\hline 5 & 57.5 & -0.183 & ONLINE-G \\
\hline
\end{tabular}

English $\rightarrow$ Czech

\begin{tabular}{rcrl} 
& Ave. $\%$ & \multicolumn{1}{c}{ Ave. $\mathrm{z}$} & System \\
\hline 1 & 67.2 & 0.594 & CUNI-TRANSFORMER \\
\hline 2 & 60.6 & 0.384 & UEDIN \\
\hline 3 & 52.1 & 0.101 & ONLINE-B \\
\hline 4 & 46.0 & -0.115 & ONLINE-A \\
\hline 5 & 42.0 & -0.246 & ONLINE-G \\
\hline
\end{tabular}

\begin{tabular}{ccrl} 
& \multicolumn{3}{c}{ German $\rightarrow$ English } \\
& Ave. \% & Ave. z & System \\
\hline 1 & 79.9 & 0.413 & RWTH \\
& 79.4 & 0.395 & UCAM \\
& 78.2 & 0.359 & NTT \\
& 77.3 & 0.346 & ONLINE-B \\
& 77.4 & 0.321 & MLLP-UPV \\
& 77.0 & 0.317 & JHU \\
& 76.9 & 0.315 & UBIQUS-NMT \\
& 76.7 & 0.310 & ONLINE-Y \\
& 75.7 & 0.268 & ONLINE-A \\
& 75.4 & 0.261 & UEDIN \\
\hline 11 & 72.5 & 0.162 & LMU-NMT \\
& 72.2 & 0.149 & NJUNMT-PRIVATE \\
\hline 13 & 65.2 & -0.074 & ONLINE-G \\
\hline 14 & 58.5 & -0.296 & ONLINE-F \\
\hline 15 & 45.4 & -0.752 & RWTH-UNSUPER \\
\hline 16 & 42.7 & -0.835 & LMU-UNSUP \\
\hline & & & \\
\hline
\end{tabular}

English $\rightarrow$ German

\begin{tabular}{rcrl} 
& Ave. $\%$ & Ave. $\mathrm{z}$ & System \\
\hline 1 & 85.5 & 0.653 & FACEBOOK-FAIR $\star$ \\
\hline 2 & 82.2 & 0.561 & ONLINE-B \\
& 81.9 & 0.551 & MICROSOFT-MARIAN \\
& 81.6 & 0.539 & MMT-PRODUCTION \\
& 82.3 & 0.537 & UCAM \\
& 80.2 & 0.491 & NTT \\
& 79.3 & 0.454 & KIT \\
\hline 8 & 77.7 & 0.396 & ONLINE-Y \\
& 76.7 & 0.377 & JHU \\
& 76.3 & 0.352 & UEDIN \\
\hline 11 & 71.8 & 0.213 & LMU-NMT \\
\hline 12 & 67.4 & 0.060 & ONLINE-A \\
\hline 13 & 53.2 & -0.385 & ONLINE-F \\
& 53.8 & -0.416 & ONLINE-G \\
\hline 15 & 36.7 & -0.966 & RWTH-UNSUPER \\
\hline 16 & 32.6 & -1.122 & LMU-UNSUP \\
\hline
\end{tabular}

\begin{tabular}{ccrl} 
& \multicolumn{3}{c}{ Estonian $\rightarrow$ English } \\
& Ave. \% & Ave. $\mathrm{z}$ & System \\
\hline 1 & 73.3 & 0.326 & TILDE-NC-NMT \\
\hline 2 & 71.1 & 0.238 & NICT \\
& 69.9 & 0.215 & TILDE-C-NMT \\
& 69.0 & 0.187 & TILDE-C-NMT-2BT \\
& 69.2 & 0.186 & UEDIN \\
& 68.7 & 0.171 & TILDE-C-NMT-COMB \\
& 67.1 & 0.117 & ONLINE-B \\
& 66.4 & 0.106 & HY-NMT \\
& 66.8 & 0.106 & TALP-UPC \\
\hline 10 & 65.4 & 0.063 & ONLINE-A \\
& 64.0 & 0.007 & CUNI-KOCMI \\
\hline 12 & 59.4 & -0.117 & NEUROTOLGE.EE \\
\hline 13 & 52.7 & -0.341 & ONLINE-G \\
\hline 14 & 34.6 & -0.950 & UNSUPTARTU \\
\hline
\end{tabular}

English $\rightarrow$ Estonian Ave. \% Ave. $\mathrm{z}$ System

\begin{tabular}{rrrl} 
& Are. $\%$ & Are. $z$ & System \\
\hline 1 & 64.9 & 0.549 & TILDE-NC-NMT \\
\hline 2 & 62.1 & 0.453 & NICT \\
& 61.6 & 0.427 & TILDE-C-NMT \\
& 61.2 & 0.418 & TILDE-C-NMT-2BT \\
\hline 5 & 58.6 & 0.340 & AALTO \\
& 58.6 & 0.329 & HY-NMT \\
& 57.5 & 0.295 & UEDIN \\
\hline 8 & 55.5 & 0.216 & CUNI-KOCMI \\
& 54.6 & 0.181 & TALP-UPC \\
\hline 10 & 52.1 & 0.097 & ONLINE-B \\
\hline 11 & 45.7 & -0.132 & NEUROTOLGE.EE \\
\hline 12 & 43.8 & -0.195 & ONLINE-A \\
\hline 13 & 37.6 & -0.406 & ONLINE-G \\
\hline 14 & 34.3 & -0.520 & PARFDA
\end{tabular}

Finnish $\rightarrow$ English

Ave. $\%$ Ave. $\mathrm{z}$ System

$\begin{array}{llll} & 75.2 & 0.153 & \text { NICT }\end{array}$

$\begin{array}{lll}74.4 & 0.128 & \text { HY-NMT }\end{array}$

$\begin{array}{lll}74.0 & 0.103 & \text { UEDIN } \\ 72.7 & 0.083 & \text { CUNI-K }\end{array}$

$\begin{array}{lll}72.7 & 0.083 & \text { CUNI-KoсMI }\end{array}$

$\begin{array}{lll}72.9 & 0.078 & \text { ONLINE-B }\end{array}$

$71.9 \quad 0.047 \quad$ TALP-UPC

$\begin{array}{lll}71.5 & 0.045 & \text { ONLINE-A }\end{array}$

\begin{tabular}{rrrr}
8 & 66.1 & -0.134 & ONLINE-G \\
\hline & 58.9 & -0.404 & JUCBNMT
\end{tabular}

$\begin{array}{llll}9 & 58.9 & -0.404 & \text { JUCBNMT }\end{array}$
English $\rightarrow$ Finnish

\begin{tabular}{ccrl} 
& \multicolumn{3}{c}{ English $\rightarrow$ Finnish } \\
& Ave. \% & Ave. z & System \\
\hline 1 & 64.7 & 0.521 & NICT \\
& 63.1 & 0.466 & HY-NMT \\
\hline 3 & 59.2 & 0.324 & UEDIN \\
& 58.3 & 0.271 & AALTO \\
& 57.9 & 0.258 & HY-NMT-2STEP \\
& 57.4 & 0.238 & TALP-UPC \\
& 55.9 & 0.184 & CUNI-KoCMI \\
& 56.6 & 0.183 & ONLINE-B \\
\hline 9 & 45.9 & -0.212 & ONLINE-A \\
& 45.3 & -0.233 & ONLINE-G \\
\hline 11 & 42.7 & -0.334 & HY-SMT \\
& 41.5 & -0.369 & HY-AH \\
\hline
\end{tabular}

Russian $\rightarrow$ English

\begin{tabular}{|c|c|c|c|}
\hline & Ave. $\%$ & Ave. $\mathrm{z}$ & System \\
\hline \multirow[t]{3}{*}{1} & 81.0 & 0.215 & ALIBABA \\
\hline & 80.3 & 0.192 & ONLINE-B \\
\hline & 79.6 & 0.170 & ONLINE-G \\
\hline 4 & 77.5 & 0.110 & UEDIN \\
\hline 5 & 76.2 & 0.034 & ONLINE-A \\
\hline \multirow[t]{3}{*}{6} & 74.1 & -0.014 & AFRL-SYSCOMB \\
\hline & 73.7 & -0.027 & JHU \\
\hline & 64.2 & -0.398 & ONLINE-F \\
\hline
\end{tabular}

English $\rightarrow$ Russian

\begin{tabular}{rcrl} 
& Ave. $\%$ & \multicolumn{1}{c}{ Ave. $\mathrm{z}$} & System \\
\hline 1 & 72.0 & 0.352 & ALIBABA-ENS \\
& 71.4 & 0.324 & ONLINE-G \\
\hline 3 & 66.8 & 0.159 & ONLINE-B \\
& 66.0 & 0.144 & UEDIN \\
& 64.9 & 0.115 & PROMT-HYB-MARIAN \\
\hline 6 & 63.9 & 0.066 & PROMT-HYB-OPENNMT \\
\hline 7 & 62.2 & -0.004 & ONLINE-A \\
\hline 8 & 59.1 & -0.075 & PROMT-RULE-BASED \\
\hline 9 & 44.5 & -0.580 & ONLINE-F \\
\hline
\end{tabular}

Turkish $\rightarrow$ English

\begin{tabular}{|c|c|c|c|}
\hline & Ave. \% & Ave. $\mathrm{z}$ & System \\
\hline \multirow[t]{6}{*}{1} & 70.2 & 0.101 & ONLINE-G \\
\hline & 69.3 & 0.077 & ONLINE-A \\
\hline & 68.1 & 0.030 & ALIBABA-ENS \\
\hline & 68.0 & 0.027 & ONLINE-B \\
\hline & 67.0 & -0.008 & UEDIN \\
\hline & 66.0 & -0.040 & NICT \\
\hline
\end{tabular}

English $\rightarrow$ Turkish

Ave. \% Ave. z System

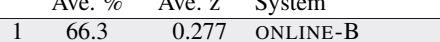
$63.6 \quad 0.222 \quad$ UEDIN

$63.5 \quad 0.216 \quad$ ALIBABA-ENS-A

$62.0 \quad 0.128 \quad$ NICT

$60.1 \quad 0.111 \quad$ ALIBABA-ENS-B

$60.1 \quad 0.058 \quad$ ONLINE-G

$\begin{array}{llll}7 & 55.0 & -0.060 & \text { RWTH }\end{array}$

$\begin{array}{llll}8 & 49.6 & -0.254 & \text { ONLINE-A }\end{array}$

Table 8: Official results of WMT18 News Translation Task. Systems ordered by standardized mean DA score, though systems within a cluster are considered tied. Lines between systems indicate clusters according to Wilcoxon rank-sum test at p-level $p<0.05$. Systems with gray background indicate use of resources that fall outside the constraints provided for the shared task. 
Costs are mounting in the case, with hundreds of pages of affidavits, emails and reports by companies including Deloitte, Pitcher Partners and Charter Keck Cramer filed and top barristers including Allan Myers, QC, and senior solicitors retained by both sides.

V tomto př́ípadě rostou Chile má deset členú a koncem srpna by měl společností, včetně společností Deloitte, Pitcher Partners a Charty Keck Cramer, a špičkových obhájců včetně Allana Myerse, QC a vyšších právních zástupců, které si ponechaly obě strany.

Figure 5: Screen shot of source-based Direct Assessment in the Appraise interface used in the English $\rightarrow$ Czech pilot campaign. The annotator is presented with a source text and a single system output randomly selected from competing systems (anonymized), and is asked to rate the translation on a sliding scale.

taken with a degree of caution for several reasons which we outline below.

Firstly, the alternate HIT structure applied in this version of DA has not been tested thoroughly enough to be certain of high reliability. For example, as described in Section 3.3, forcing all translations of a given source segment to be assessed by the same human judge within the same HIT could cause individual DA ratings to become highly relative as opposed to the aim of DA ratings to be as close as possible to absolute judgments of translation quality. Furthermore, an additional bias that could cause problems for this HIT structure is one associated with a past evaluation method, relative ranking. When evaluating competing translations of the same source that are situated in close proximity within a HIT, annotators may be primed by high (or low) quality outputs resulting in overly severe (or lenient) judgments for subsequent translations of the same source segment (Bojar et al., 2011).

Secondly, while standard monolingual DA employs annotators only required to be speakers of a single language, source-based DA requires fluency in two languages and it is not known the degree to which varying levels of native language fluency in at least one language may negatively impact the reliability of DA rankings in the case of bilingual annotators.

Thirdly, it is likely that the quality of reference translations can vary and this could potentially impact the reliability of human performance estimates in source-based DA. Although reference- based DA assumes high quality reference translations, in the unfortunate case of problematic references, the overall rankings are unlikely to suffer to any large degree in terms of the reliability of system rankings, since all competing systems are likely to suffer equally from any lack of quality in reference translations.

However, in the adapted source-based version of DA, the effect of low quality reference translations is quite different. Firstly, since assessment involves comparison of MT outputs with the source, genuine participating systems will not suffer from the fact reference translations are low quality, since references are not involved in their evaluation. On the other hand, human performance estimates certainly will, as a drop in reference quality is indeed highly likely to negatively impact the placement of human performance estimates in system rankings. The reliability of comparisons with human performance with sourcebased DA is therefore highly dependent on high quality reference translations, as employment of a low quality set of references can only lead to underestimates of human performance. Considering the manual evaluation included several reports of ill-formed reference translations, conclusions of human parity and/or superiority relative to humans should be avoided.

\subsection{Considerations as to Human Parity}

As mentioned above, before making any statements about "machine translation outperforming humans" or "machine-human parity in translation" it may be important to consider the following ad- 


\begin{tabular}{rrrl} 
& & \multicolumn{2}{c}{ English $\rightarrow$ Czech } \\
& Ave. $\%$ & Ave. $\mathrm{z}$ & System \\
\hline 1 & 84.4 & 0.667 & CUNI-TRANSFORMER \\
\hline 2 & 79.8 & 0.521 & UEDIN \\
& 78.6 & 0.483 & NEWSTEST2018-REF \\
\hline 4 & 68.1 & 0.128 & ONLINE-B \\
\hline 5 & 59.4 & -0.178 & ONLINE-A \\
\hline 6 & 54.1 & -0.354 & ONLINE-G \\
\hline
\end{tabular}

Table 9: Source-based DA results for English $\rightarrow$ Czech newstest2018, where systems are ordered by standardized mean DA score, though systems within a cluster are considered tied. Lines between systems indicate clusters according to Wilcoxon rank-sum test at $\mathrm{p}$-level $p<0.05$. Systems with gray background indicate use of resources that fall outside the constraints provided for the shared task. NEWSTEST2018-REF denotes the human system comprised of human-produced reference translations.

ditional points:

- Since none of WMT18 systems process larger units than individual sentences and our evaluation does not include any context beyond individual segments, it is possible that the human estimate is under-rewarded for correct cross-sentential phenomena.

- The sample size employed in the sourcebased DA evaluation was smaller than the recommended 1,500 judgments per system.

- The way in which translations in the test sets were originally created was as follows: one half of the test data for a given language pair was translated in one language direction and the other half in the opposite direction. It is well known that the translation direction affects translation quality in training and this could also be the case for evaluation.

- The formal education in linguistics or translatology of human assessors has not been taken into account: it is likely that whether or not human assessors have received any formal training in translation might influence their acceptance of varying levels of wellformedness in translations. For example, untrained assessors might not be as sensitive to subtle differences in verb conjugation, based on their own experience: In many real-life situations, the exact verb tense or conditional chosen in one sentence may not really impact the overall message because it can be implied from the context (and thus left free to the imagination of the annotator in our sentence-based evaluation) or from general knowledge.

\section{Test Suites}

Arguably, both the manual and automatic evaluations carried out at WMT News Translation Task are rather opaque. We learn (for each language pair and with a known confidence) which systems perform better on average over the sentences sampled from the news test set.

This average performance however does not provide any insight into which particular phenomena are handled better or worse by the systems. It is quite possible that the overall best-performing system may be unreliable for long sentences, for named entities, for pronouns or others. Such targeted evaluations may be important for particular deployment settings and use cases, and they are definitely important for us, MT system developers, in order to focus on them in subsequent research.

\section{Acknowledgments}

This work was supported in part by funding from the European Union's Horizon 2020 research and innovation programme under grant agreement Nos. 645452 (QT21) and 645357 (Cracker), and from the Connecting Europe Facility under agreement No. NEA/CEF/ICT/A2016/1331648 (ParaCrawl).

We would also like to thank the University of Helsinki, the University of Tartu, Yandex and Microsoft for supplying test data for the news translation task.

The human evaluation campaign was very gratefully supported by contributions from Amazon, Microsoft, and Science Foundation Ireland in the ADAPT Centre for Digital Content Technology (www.adaptcentre.ie) at Dublin City University funded under the SFI Research Centres Programme (Grant 13/RC/2106) co-funded under the European Regional Development Fund.

We would also like to give special thanks to the small group of Turkish speakers who rescued our English-Turkish human evaluation at very short notice by contributing their time voluntarily. Finally, we are grateful to the large number of anonymous Mechanical Turk workers who contributed their human intelligence to the human evaluation.

\section{References}

Mikel Artetxe, Gorka Labaka, Eneko Agirre, and Kyunghyun Cho. 2018. Unsupervised neural ma- 
chine translation. In Proceedings of the Sixth International Conference on Learning Representations.

Dzmitry Bahdanau, Kyunghyun Cho, and Yoshua Bengio. 2014. Neural Machine Translation by Jointly Learning to Align and Translate. arXiv e-prints, abs/1409.0473. Presented at ICLR 2015.

Loïc Barrault, Fethi Bougares, Lucia Specia, Chiraag Lala, Desmond Elliott, and Stella Frank. 2018. Findings of the Third Shared Task on Multimodal Machine Translation. In Proceedings of the Third Conference on Machine Translation, Brussels, Belgium. Association for Computational Linguistics.

Chao Bei, Hao Zong, Yiming Wang, Baoyong Fan, Shiqi Li, and Conghu Yuan. 2018. An Empirical Study of Machine Translation for the Shared Task of WMT18. In Proceedings of the Third Conference on Machine Translation, Brussels, Belgium. Association for Computational Linguistics.

Ergun Biçici. 2018. Robust parfda Statistical Machine Translation Results. In Proceedings of the Third Conference on Machine Translation, Brussels, Belgium. Association for Computational Linguistics.

Ondrej Bojar, Christian Buck, Christian Federmann, Barry Haddow, Philipp Koehn, Johannes Leveling, Christof Monz, Pavel Pecina, Matt Post, Herve Saint-Amand, Radu Soricut, Lucia Specia, and Aleš Tamchyna. 2014. Findings of the 2014 Workshop on Statistical Machine Translation. In Proceedings of the Ninth Workshop on Statistical Machine Translation, pages 12-58, Baltimore, Maryland, USA. Association for Computational Linguistics.

Ondřej Bojar, Rajen Chatterjee, Christian Federmann, Yvette Graham, Barry Haddow, Matthias Huck, Philipp Koehn, Varvara Logacheva, Christof Monz, Matteo Negri, Matt Post, Raphael Rubino, Lucia Specia, and Marco Turchi. 2017. Findings of the 2017 Conference on Machine Translation (WMT17). In Proceedings of the Second Conference on Machine Translation, Copenhagen, Denmark. Association for Computational Linguistics.

Ondřej Bojar, Miloš Ercegovčević, Martin Popel, and Omar Zaidan. 2011. A Grain of Salt for the WMT Manual Evaluation. In Proceedings of the Sixth Workshop on Statistical Machine Translation, pages 1-11, Edinburgh, Scotland. Association for Computational Linguistics.

Ondřej Bojar, Christian Buck, Chris Callison-Burch, Christian Federmann, Barry Haddow, Philipp Koehn, Christof Monz, Matt Post, Radu Soricut, and Lucia Specia. 2013. Findings of the 2013 Workshop on Statistical Machine Translation. In Proceedings of the Eighth Workshop on Statistical Machine Translation, pages 1-44, Sofia, Bulgaria. Association for Computational Linguistics.

Ondřej Bojar, Rajen Chatterjee, Christian Federmann, Yvette Graham, Barry Haddow, Matthias Huck,
Antonio Jimeno Yepes, Philipp Koehn, Varvara Logacheva, Christof Monz, Matteo Negri, Aurelie Neveol, Mariana Neves, Martin Popel, Matt Post, Raphael Rubino, Carolina Scarton, Lucia Specia, Marco Turchi, Karin Verspoor, and Marcos Zampieri. 2016a. Findings of the 2016 Conference on Machine Translation. In Proceedings of the First Conference on Machine Translation, pages 131-198, Berlin, Germany. Association for Computational Linguistics.

Ondřej Bojar, Rajen Chatterjee, Christian Federmann, Barry Haddow, Matthias Huck, Chris Hokamp, Philipp Koehn, Varvara Logacheva, Christof Monz, Matteo Negri, Matt Post, Carolina Scarton, Lucia Specia, and Marco Turchi. 2015. Findings of the 2015 Workshop on Statistical Machine Translation. In Proceedings of the Tenth Workshop on Statistical Machine Translation, pages 1-46, Lisbon, Portugal. Association for Computational Linguistics.

Ondřej Bojar, Ondřej Dušek, Tom Kocmi, Jindřich Libovický, Michal Novák, Martin Popel, Roman Sudarikov, and Dušan Variš. 2016b. CzEng 1.6: Enlarged Czech-English Parallel Corpus with Processing Tools Dockered. In Text, Speech and Dialogue: 19th International Conference, TSD 2016, Brno, Czech Republic, September 12-16, 2016, Proceedings. Springer Verlag.

Ondřej Bojar, Jiří Mírovský, Kateřina Rysová, and Magdaléna Rysová. 2018. EvalD Reference-Less Discourse Evaluation for WMT18. In Proceedings of the Third Conference on Machine Translation, Brussels, Belgium. Association for Computational Linguistics.

Franck Burlot, Yves Scherrer, Vinit Ravishankar, Ondřej Bojar, Stig-Arne Grönroos, Maarit Koponen, Tommi Nieminen, and François Yvon. 2018. The WMT'18 Morpheval test suites for English-Czech, English-German, English-Finnish and Turkish-English. In Proceedings of the Third Conference on Machine Translation, Brussels, Belgium. Association for Computational Linguistics.

Chris Callison-Burch, Cameron Shaw Fordyce, Philipp Koehn, Christof Monz, and Josh Schroeder. 2007. (Meta-) Evaluation of Machine Translation. In Proceedings of the Second Workshop on Statistical Machine Translation, pages 136-158, Prague, Czech Republic. Association for Computational Linguistics.

Chris Callison-Burch, Cameron Shaw Fordyce, Philipp Koehn, Christof Monz, and Josh Schroeder. 2008. Further Meta-Evaluation of Machine Translation. In Proceedings of the Third Workshop on Statistical Machine Translation, pages 70-106, Columbus, Ohio. Association for Computational Linguistics.

Chris Callison-Burch, Philipp Koehn, Christof Monz, Kay Peterson, Mark Przybocki, and Omar Zaidan. 2010. Findings of the 2010 Joint Workshop on Statistical Machine Translation and Metrics for Machine Translation. In Proceedings of the Joint Fifth 
Workshop on Statistical Machine Translation and MetricsMATR, pages 17-53, Uppsala, Sweden. Association for Computational Linguistics.

Chris Callison-Burch, Philipp Koehn, Christof Monz, Matt Post, Radu Soricut, and Lucia Specia. 2012. Findings of the 2012 Workshop on Statistical Machine Translation. In Proceedings of the Seventh Workshop on Statistical Machine Translation, pages 10-48, Montreal, Canada. Association for Computational Linguistics.

Chris Callison-Burch, Philipp Koehn, Christof Monz, and Josh Schroeder. 2009. Findings of the 2009 Workshop on Statistical Machine Translation. In Proceedings of the Fourth Workshop on Statistical Machine Translation, pages 1-28, Athens, Greece. Association for Computational Linguistics.

Chris Callison-Burch, Philipp Koehn, Christof Monz, and Omar Zaidan. 2011. Findings of the 2011 Workshop on Statistical Machine Translation. In Proceedings of the Sixth Workshop on Statistical Machine Translation, pages 22-64, Edinburgh, Scotland. Association for Computational Linguistics.

Noe Casas, Carlos Escolano, Marta R. Costa-jussà, and José A. R. Fonollosa. 2018. The TALP-UPC Machine Translation Systems for WMT18 News Shared Translation Task. In Proceedings of the Third Conference on Machine Translation, Brussels, Belgium. Association for Computational Linguistics.

Rajen Chatterjee, Matteo Negri, Raphael Rubino, and Marco Turchi. 2018. Findings of the WMT 2018 Shared Task on Automatic Post-Editing. In Proceedings of the Third Conference on Machine Translation, Brussels, Belgium. Association for Computational Linguistics.

Alexis Conneau, Guillaume Lample, Marc'Aurelio Ranzato, Ludovic Denoyer, and Hervé Jégou. 2017. Word Translation Without Parallel Data. CoRR, abs/1710.04087.

Maksym Del, Andre Tättar, and Mark Fishel. 2018. Phrase-based Unsupervised Machine Translation with Compositional Phrase Embeddings. In Proceedings of the Third Conference on Machine Translation, Brussels, Belgium. Association for Computational Linguistics.

Yongchao Deng, Shanbo Cheng, Jun Lu, Kai Song, Jingang Wang, Shenglan Wu, Liang Yao, Guchun Zhang, Haibo Zhang, Pei Zhang, Changfeng Zhu, and Boxing Chen. 2018. Alibaba's Neural Machine Translation Systems for WMT18. In Proceedings of the Third Conference on Machine Translation, Brussels, Belgium. Association for Computational Linguistics.

Sergey Edunov, Myle Ott, Michael Auli, and David Grangier. 2018. Understanding Back-Translation at Scale. In Proceedings of the 2018 Conference on Empirical Methods in Natural Language Processing
(EMNLP), Brussels, Belgium. Association for Computational Linguistics.

Christian Federmann. 2012. Appraise: an open-source toolkit for manual evaluation of $\mathrm{mt}$ output. The Prague Bulletin of Mathematical Linguistics, 98:2535.

Markus Freitag, Minwei Feng, Matthias Huck, Stephan Peitz, and Hermann Ney. 2013. Reverse Word Order Models. In Proceedings of the XIV Machine Translation Summit, pages 159-166, Nice, France.

Markus Freitag, Matthias Huck, and Hermann Ney. 2014. Jane: Open Source Machine Translation System Combination. In Proceedings of the Demonstrations at the 14th Conference of the European Chapter of the Association for Computational Linguistics, pages 29-32, Gothenburg, Sweden. Association for Computational Linguistics.

Adrià de Gispert, Bill Byrne, Eva Hasler, and Felix Stahlberg. 2017. Neural machine translation by minimising the bayes-risk with respect to syntactic translation lattices. In Proceedings of the 15th Conference of the European Chapter of the Association for Computational Linguistics, EACL 2017, Valencia, Spain, April 3-7, 2017, Volume 2: Short Papers, pages 362-368.

Yvette Graham, Timothy Baldwin, Alistair Moffat, and Justin Zobel. 2013. Continuous Measurement Scales in Human Evaluation of Machine Translation. In Proceedings of the 7th Linguistic Annotation Workshop and Interoperability with Discourse, pages 33-41, Sofia, Bulgaria. Association for Computational Linguistics.

Yvette Graham, Timothy Baldwin, Alistair Moffat, and Justin Zobel. 2014. Is Machine Translation Getting Better over Time? In Proceedings of the 14th Conference of the European Chapter of the Association for Computational Linguistics, pages 443451, Gothenburg, Sweden. Association for Computational Linguistics.

Yvette Graham, Timothy Baldwin, Alistair Moffat, and Justin Zobel. 2016. Can machine translation systems be evaluated by the crowd alone. Natural Language Engineering, pages 1-28.

Miguel Graça, Yunsu Kim, Julian Schamper, Jiahui Geng, and Hermann Ney. 2018. The RWTH Aachen University English-German and GermanEnglish Unsupervised Neural Machine Translation Systems for WMT 2018. In Proceedings of the Third Conference on Machine Translation, Brussels, Belgium. Association for Computational Linguistics.

Stig-Arne Grönroos, Sami Virpioja, and Mikko Kurimo. 2018. Cognate-aware morphological segmentation for multilingual neural translation. In Proceedings of the Third Conference on Machine Translation, Brussels, Belgium. Association for Computational Linguistics. 
Jeremy Gwinnup, Tim Anderson, Grant Erdmann, and Katherine Young. 2018. The AFRL WMT18 Systems: Ensembling, Continuation and Combination. In Proceedings of the Third Conference on Machine Translation, Brussels, Belgium. Association for Computational Linguistics.

Jeremy Gwinnup, Timothy Anderson, Grant Erdmann, Katherine Young, Michaeel Kazi, Elizabeth Salesky, Brian Thompson, and Jonathan Taylor. 2017. The AFRL-MITLL WMT17 Systems: Old, New, Borrowed, BLEU. In Proceedings of the Second Conference on Machine Translation, Copenhagen, Denmark. Association for Computational Linguistics.

Barry Haddow, Nikolay Bogoychev, Denis Emelin, Ulrich Germann, Roman Grundkiewicz, Kenneth Heafield, Antonio Valerio Miceli Barone, and Rico Sennrich. 2018. The University of Edinburgh's Submissions to the WMT18 News Translation Task. In Proceedings of the Third Conference on Machine Translation, Brussels, Belgium. Association for Computational Linguistics.

Hany Hassan, Anthony Aue, Chang Chen, Vishal Chowdhary, Jonathan Clark, Christian Federmann, Xuedong Huang, Marcin Junczys-Dowmunt, William Lewis, Mu Li, Shujie Liu, Tie-Yan Liu, Renqian Luo, Arul Menezes, Tao Qin, Frank Seide, Xu Tan, Fei Tian, Lijun Wu, Shuangzhi Wu, Yingce Xia, Dongdong Zhang, Zhirui Zhang, and Ming Zhou. 2018. Achieving Human Parity on Automatic Chinese to English News Translation. https://www.microsoft.com/ en-us/research/uploads/prod/2018/03/ final-achieving-human.pdf.

Kenneth Heafield. 2011. KenLM: Faster and Smaller Language Model Queries. In Proceedings of the Sixth Workshop on Statistical Machine Translation, pages 187-197, Edinburgh, Scotland. Association for Computational Linguistics.

Felix Hieber, Tobias Domhan, Michael Denkowski, David Vilar, Artem Sokolov, Ann Clifton, and Matt Post. 2017. Sockeye: A Toolkit for Neural Machine Translation. arXiv preprint arXiv:1712.05690.

Bojie Hu, Ambyer Han, and Shen Huang. 2018. TencentFmRD Neural Machine Translation for WMT18. In Proceedings of the Third Conference on Machine Translation, Brussels, Belgium. Association for Computational Linguistics.

Matthias Huck, Fabienne Braune, and Alexander Fraser. 2017a. LMU Munich's Neural Machine Translation Systems for News Articles and Health Information Texts. In Proceedings of the Second Conference on Machine Translation, Copenhagen, Denmark. Association for Computational Linguistics.

Matthias Huck, Simon Riess, and Alexander Fraser. 2017b. Target-side Word Segmentation Strategies for Neural Machine Translation. In Proceedings of the Second Conference on Machine Translation, Copenhagen, Denmark. Association for Computational Linguistics.

Matthias Huck, Dario Stojanovski, Viktor Hangya, and Alexander Fraser. 2018. LMU Munich's Neural Machine Translation Systems at WMT 2018. In Proceedings of the Third Conference on Machine Translation, Brussels, Belgium. Association for Computational Linguistics.

Arvi Hurskainen and Jörg Tiedemann. 2017. Rulebased Machine translation from English to Finnish. In Proceedings of the Second Conference on Machine Translation, Copenhagen, Denmark. Association for Computational Linguistics.

Javier Iranzo-Sánchez, Pau Baquero-Arnal, Gonçal V. Garcés Díaz-Munío, Adrià Martínez-Villaronga, Jorge Civera, and Alfons Juan. 2018. The MLLPUPV German-English Machine Translation System for WMT18. In Proceedings of the Third Conference on Machine Translation, Brussels, Belgium. Association for Computational Linguistics.

Marcin Junczys-Dowmunt. 2018. Microsoft's Submission to the WMT2018 News Translation Task: How I Learned to Stop Worrying and Love the Data. In Proceedings of the Third Conference on Machine Translation, Brussels, Belgium. Association for Computational Linguistics.

Marcin Junczys-Dowmunt, Roman Grundkiewicz, Tomasz Dwojak, Hieu Hoang, Kenneth Heafield, Tom Neckermann, Frank Seide, Ulrich Germann, Alham Fikri Aji, Nikolay Bogoychev, André F. T. Martins, and Alexandra Birch. 2018. Marian: Fast neural machine translation in c++. In Proceedings of ACL 2018, System Demonstrations, pages 116-121. Association for Computational Linguistics.

Guillaume Klein, Yoon Kim, Yuntian Deng, Jean Senellart, and Alexander Rush. 2017. OpenNMT: Open-Source Toolkit for Neural Machine Translation. In Proceedings of ACL 2017, System Demonstrations, pages 67-72, Vancouver, Canada. Association for Computational Linguistics.

Tom Kocmi, Roman Sudarikov, and Ondřej Bojar. 2018. CUNI Submissions in WMT18. In Proceedings of the Third Conference on Machine Translation, Brussels, Belgium. Association for Computational Linguistics.

Philipp Koehn, Kevin Duh, and Brian Thompson. 2018a. The JHU Machine Translation Systems for WMT 2018. In Proceedings of the Third Conference on Machine Translation, Brussels, Belgium. Association for Computational Linguistics.

Philipp Koehn, Hieu Hoang, Alexandra Birch, Chris Callison-Burch, Marcello Federico, Nicola Bertoldi, Brooke Cowan, Wade Shen, Christine Moran, Richard Zens, Christopher J. Dyer, Ondřej Bojar, Alexandra Constantin, and Evan Herbst. 2007. 
Moses: Open Source Toolkit for Statistical Machine Translation. In Proceedings of the 45th Annual Meeting of the Association for Computational Linguistics Companion Volume Proceedings of the Demo and Poster Sessions, pages 177-180, Prague, Czech Republic. Association for Computational Linguistics.

Philipp Koehn, Huda Khayrallah, Kenneth Heafield, and Mikel L. Forcada. 2018b. Findings of the WMT 2018 Shared Task on Parallel Corpus Filtering. In Proceedings of the Third Conference on Machine Translation, Brussels, Belgium. Association for Computational Linguistics.

Philipp Koehn and Christof Monz. 2006. Manual and Automatic Evaluation of Machine Translation between European Languages. In Proceedings on the Workshop on Statistical Machine Translation, pages 102-121, New York City. Association for Computational Linguistics.

Guillaume Lample, Myle Ott, Alexis Conneau, Ludovic Denoyer, and Marc'Aurelio Ranzato. 2018. Phrase-Based \& Neural Unsupervised Machine Translation. arXiv preprint arXiv:1804.07755.

Lemao Liu, Masao Utiyama, Andrew Finch, and Eiichiro Sumita. 2016. Agreement on Targetbidirectional Neural Machine Translation. In Proceedings of the 2016 Conference of the North American Chapter of the Association for Computational Linguistics: Human Language Technologies, pages 411-416, San Diego, CA, USA. Association for Computational Linguistics.

Qingsong Ma, Ondřej Bojar, and Yvette Graham. 2018. Results of the WMT18 metrics shared task: Both characters and embeddings achieve good performance. In Proceedings of the Third Conference on Machine Translation, Volume 2: Shared Task Papers, Brussels, Belgium. Association for Computational Linguistics.

Sainik Kumar Mahata, Dipankar Das, and Sivaji Bandyopadhyay. 2018. JUCBNMT at WMT2018 News Translation Task: Character Based Neural Machine Translation of Finnish to English. In Proceedings of the Third Conference on Machine Translation, Brussels, Belgium. Association for Computational Linguistics.

Benjamin Marie, Rui Wang, Atsushi Fujita, Masao Utiyama, and Eiichiro Sumita. 2018. NICT's Neural and Statistical Machine Translation Systems for the WMT18 News Translation Task. In Proceedings of the Third Conference on Machine Translation, Brussels, Belgium. Association for Computational Linguistics.

Alexander Molchanov. 2018. PROMT Systems for WMT 2018 Shared Translation Task. In Proceedings of the Third Conference on Machine Translation, Brussels, Belgium. Association for Computational Linguistics.
Makoto Morishita, Jun Suzuki, and Masaaki Nagata. 2018. NTT's Neural Machine Translation Systems for WMT 2018. In Proceedings of the Third Conference on Machine Translation, Brussels, Belgium. Association for Computational Linguistics.

Mariana Neves, Antonio Jimeno Yepes, Aurélie Névéol, Cristian Grozea, Amy Siu, Madeleine Kittner, and Karin Verspoor. 2018. Findings of the WMT 2018 Biomedical Translation Shared Task: Evaluation on Medline test sets. In Proceedings of the Third Conference on Machine Translation, Brussels, Belgium. Association for Computational Linguistics.

Ngoc-Quan Pham, Jan Niehues, and Alexander Waibel. 2018. The Karlsruhe Institute of Technology Systems for the News Translation Task in WMT 2018. In Proceedings of the Third Conference on Machine Translation, Brussels, Belgium. Association for Computational Linguistics.

Mārcis Pinnis, Matīss Rikters, and Rihards Krišlauks. 2018. Tilde's Machine Translation Systems for WMT 2018. In Proceedings of the Third Conference on Machine Translation, Brussels, Belgium. Association for Computational Linguistics.

Martin Popel. 2018. CUNI Transformer Neural MT System for WMT18. In Proceedings of the Third Conference on Machine Translation, Brussels, Belgium. Association for Computational Linguistics.

Martin Popel and Ondřej Bojar. 2018. Training Tips for the Transformer Model. The Prague Bulletin of Mathematical Linguistics, 110(1):43-70.

Alessandro Raganato, Yves Scherrer, Tommi Nieminen, Arvi Hurskainen, and Jörg Tiedemann. 2018. The University of Helsinki submissions to the WMT18 news task. In Proceedings of the Third Conference on Machine Translation, Brussels, Belgium. Association for Computational Linguistics.

Julian Schamper, Jan Rosendahl, Parnia Bahar, Yunsu Kim, Arne Nix, and Hermann Ney. 2018. The RWTH Aachen University Supervised Machine Translation Systems for WMT 2018. In Proceedings of the Third Conference on Machine Translation, Brussels, Belgium. Association for Computational Linguistics.

Rico Sennrich, Orhan Firat, Kyunghyun Cho, Alexandra Birch, Barry Haddow, Julian Hitschler, Marcin Junczys-Dowmunt, Samuel Läubli, Antonio Valerio Miceli Barone, Jozef Mokry, and Maria Nadejde. 2017. Nematus: a Toolkit for Neural Machine Translation. In Proceedings of the Software Demonstrations of the 15th Conference of the European Chapter of the Association for Computational Linguistics, pages 65-68, Valencia, Spain. Association for Computational Linguistics.

Rico Sennrich, Barry Haddow, and Alexandra Birch. 2016. Neural Machine Translation of Rare Words 
with Subword Units. In Proceedings of the 54th Annual Meeting of the Association for Computational Linguistics (Volume 1: Long Papers), pages 17151725, Berlin, Germany. Association for Computational Linguistics.

Lucia Specia, Frédéric Blain, Varvara Logacheva, Ramón Astudillo, and André F. T. Martins. 2018. Findings of the WMT 2018 Shared Task on Quality Estimation. In Proceedings of the Third Conference on Machine Translation, Brussels, Belgium. Association for Computational Linguistics.

Felix Stahlberg, Adrià de Gispert, and Bill Byrne. 2018. The University of Cambridge's Machine Translation Systems for WMT18. In Proceedings of the Third Conference on Machine Translation, Brussels, Belgium. Association for Computational Linguistics.

Dario Stojanovski, Viktor Hangya, Matthias Huck, and Alexander Fraser. 2018. The LMU Munich Unsupervised Machine Translation Systems. In Proceedings of the Third Conference on Machine Translation, Brussels, Belgium. Association for Computational Linguistics.

Roman Sudarikov, Martin Popel, Ondřej Bojar, Aljoscha Burchardt, and Ondřej Klejch. 2016. Using MT-ComparEval. In Translation Evaluation: From Fragmented Tools and Data Sets to an Integrated Ecosystem, pages 76-82.

Sander Tars and Mark Fishel. 2018. Multi-Domain Neural Machine Translation. In Proceedings of EAMT, pages 259-268.

Jörg Tiedemann, Fabienne Cap, Jenna Kanerva, Filip Ginter, Sara Stymne, Robert Östling, and Marion Weller-Di Marco. 2016. Phrase-Based SMT for Finnish with More Data, Better Models and Alternative Alignment and Translation Tools. In Proceedings of the First Conference on Machine Translation, pages 391-398, Berlin, Germany. Association for Computational Linguistics.

Ashish Vaswani, Samy Bengio, Eugene Brevdo, Francois Chollet, Aidan N. Gomez, Stephan Gouws, Llion Jones, Łukasz Kaiser, Nal Kalchbrenner, Niki Parmar, Ryan Sepassi, Noam Shazeer, and Jakob Uszkoreit. 2018. Tensor2Tensor for Neural Machine Translation. CoRR, abs/1803.07416.

Ashish Vaswani, Noam Shazeer, Niki Parmar, Jakob Uszkoreit, Llion Jones, Aidan N Gomez, Ł ukasz Kaiser, and Illia Polosukhin. 2017. Attention is All you Need. In I. Guyon, U. V. Luxburg, S. Bengio, H. Wallach, R. Fergus, S. Vishwanathan, and R. Garnett, editors, Advances in Neural Information Processing Systems 30, pages 5998-6008. Curran Associates, Inc.

Sami Virpioja, Peter Smit, Stig-Arne Grönroos, and Mikko Kurimo. 2013. Morfessor 2.0: Python Implementation and Extensions for Morfessor Baseline. Technical report.
Mingxuan Wang, Li Gong, Wenhuan Zhu, Jun Xie, and Chao Bian. 2018a. Tencent Neural Machine Translation Systems for WMT18. In Proceedings of the Third Conference on Machine Translation, Brussels, Belgium. Association for Computational Linguistics.

Qiang Wang, Bei Li, Jiqiang Liu, Bojian Jiang, Zheyang Zhang, Yinqiao Li, Ye Lin, Tong Xiao, and Jingbo Zhu. 2018b. The NiuTrans Machine Translation System for WMT18. In Proceedings of the Third Conference on Machine Translation, Brussels, Belgium. Association for Computational Linguistics.

Yonghui Wu, Mike Schuster, Zhifeng Chen, Quoc V. Le, Mohammad Norouzi, Wolfgang Macherey, Maxim Krikun, Yuan Cao, Qin Gao, Klaus Macherey, Jeff Klingner, Apurva Shah, Melvin Johnson, Xiaobing Liu, Lukasz Kaiser, Stephan Gouws, Yoshikiyo Kato, Taku Kudo, Hideto Kazawa, Keith Stevens, George Kurian, Nishant Patil, Wei Wang, Cliff Young, Jason Smith, Jason Riesa, Alex Rudnick, Oriol Vinyals, Greg Corrado, Macduff Hughes, and Jeffrey Dean. 2016. Google's neural machine translation system: Bridging the gap between human and machine translation. CoRR, abs/1609.08144.

Weijia Xu and Marine Carpuat. 2018. The University of Maryland's Chinese-English Neural Machine Translation Systems at WMT18. In Proceedings of the Third Conference on Machine Translation, Brussels, Belgium. Association for Computational Linguistics. 


\section{A Differences in Human Scores}

Tables 10-23 show differences in average standardized human scores for all pairs of competing systems for each language pair. The numbers in each of the tables' cells indicate the difference in average standardized human scores for the system in that column and the system in that row.

Because there were so many systems and data conditions the significance of each pairwise comparison needs to be quantified. We applied Wilcoxon rank-sum test to measure the likelihood that such differences could occur simply by chance. In the following tables $\star$ indicates statistical significance at $p<0.05$, $\dagger$ indicates statistical significance at $p<0.01$, and $\ddagger$ indicates statistical significance at $p<0.001$, according to Wilcoxon rank-sum test.

Each table contains final rows showing the average score achieved by that system and the rank range according according to Wilcoxon rank-sum test $(p<0.05)$. Gray lines separate clusters based on nonoverlapping rank ranges.

Table 24 shows the differences in average standardized human scores for Czech $\rightarrow$ English systems, based on source-based DA.

\begin{tabular}{|c|c|c|c|c|c|c|c|c|c|c|c|c|c|c|}
\hline & 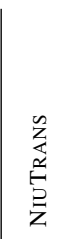 & $\begin{array}{l}\frac{m}{1} \\
\text { 岁 } \\
\frac{3}{3} \\
0\end{array}$ & 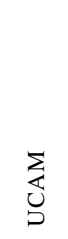 & 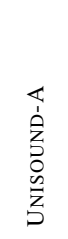 & 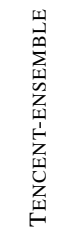 & $\begin{array}{l}\infty \\
\dot{1} \\
z \\
0 \\
o \\
\vdots \\
b\end{array}$ & 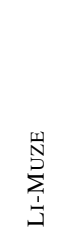 & 㫐 & 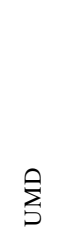 & 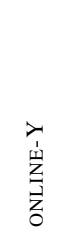 & $\begin{array}{l}\text { za } \\
\text { 离 }\end{array}$ & 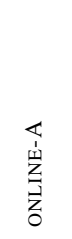 & 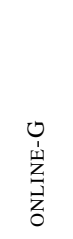 & $\begin{array}{l}\frac{1}{1} \\
\frac{1}{\mathrm{~L}} \\
\frac{1}{Z} \\
\frac{1}{0}\end{array}$ \\
\hline NiUTRANS & - & 0.03 & 0.03 & 0.03 & $0.04 \star$ & $0.05 \star$ & $0.05 \star$ & $0.05 \star$ & $0.06 \dagger$ & $0.15 \ddagger$ & $0.16 \ddagger$ & $0.20 \ddagger$ & $0.47 \ddagger$ & $0.52 \ddagger$ \\
\hline ONLINE-B & -0.03 & - & 0.00 & 0.00 & 0.01 & 0.02 & 0.02 & 0.02 & 0.03 & $0.12 \ddagger$ & $0.13 \ddagger$ & $0.17 \ddagger$ & $0.44 \ddagger$ & $0.49 \ddagger$ \\
\hline UCAM & -0.03 & 0.00 & - & 0.00 & 0.01 & 0.02 & 0.02 & 0.02 & 0.03 & $0.11 \ddagger$ & $0.13 \ddagger$ & $0.17 \ddagger$ & $0.44 \ddagger$ & $0.49 \ddagger$ \\
\hline UNISOUND-A & -0.03 & 0.00 & 0.00 & - & 0.01 & 0.01 & 0.02 & 0.02 & 0.03 & $0.11 \ddagger$ & $0.12 \ddagger$ & $0.17 \ddagger$ & $0.43 \ddagger$ & $0.48 \ddagger$ \\
\hline TENCENT-ENSEMBLE & -0.04 & -0.01 & -0.01 & -0.01 & - & 0.01 & 0.01 & 0.01 & 0.02 & $0.10 \ddagger$ & $0.12 \ddagger$ & $0.16 \ddagger$ & $0.43 \ddagger$ & $0.48 \ddagger$ \\
\hline UNISOUND-B & -0.05 & -0.02 & -0.02 & -0.01 & -0.01 & - & 0.00 & 0.01 & 0.02 & $0.10 \ddagger$ & $0.11 \ddagger$ & $0.16 \ddagger$ & $0.42 \ddagger$ & $0.47 \ddagger$ \\
\hline LI-MUZE & -0.05 & -0.02 & -0.02 & -0.02 & -0.01 & 0.00 & - & 0.00 & 0.01 & $0.10 \ddagger$ & $0.11 \ddagger$ & $0.15 \ddagger$ & $0.42 \ddagger$ & $0.47 \ddagger$ \\
\hline NICT & -0.05 & -0.02 & -0.02 & -0.02 & -0.01 & -0.01 & 0.00 & - & 0.01 & $0.09 \ddagger$ & $0.11 \ddagger$ & $0.15 \ddagger$ & $0.42 \ddagger$ & $0.47 \ddagger$ \\
\hline UMD & -0.06 & -0.03 & -0.03 & -0.03 & -0.02 & -0.02 & -0.01 & -0.01 & - & $0.08 \dagger$ & $0.10 \ddagger$ & $0.14 \ddagger$ & $0.40 \ddagger$ & $0.45 \ddagger$ \\
\hline ONLINE-Y & -0.15 & -0.12 & -0.11 & -0.11 & -0.10 & -0.10 & -0.10 & -0.09 & -0.08 & - & 0.01 & $0.06 \star$ & $0.32 \ddagger$ & $0.37 \ddagger$ \\
\hline UEDIN & -0.16 & -0.13 & -0.13 & -0.12 & -0.12 & -0.11 & -0.11 & -0.11 & -0.10 & -0.01 & - & $0.04 \star$ & $0.31 \ddagger$ & $0.36 \ddagger$ \\
\hline ONLINE-A & -0.20 & -0.17 & -0.17 & -0.17 & -0.16 & -0.16 & -0.15 & -0.15 & -0.14 & -0.06 & -0.04 & - & $0.27 \ddagger$ & $0.32 \ddagger$ \\
\hline ONLINE-G & -0.47 & -0.44 & -0.44 & -0.43 & -0.43 & -0.42 & -0.42 & -0.42 & -0.40 & -0.32 & -0.31 & -0.27 & - & $0.05 \star$ \\
\hline ONLINE-F & -0.52 & -0.49 & -0.49 & -0.48 & -0.48 & -0.47 & -0.47 & -0.47 & -0.45 & -0.37 & -0.36 & -0.32 & -0.05 & - \\
\hline & 0.14 & 0.11 & 0.11 & 0.11 & 0.10 & 0.09 & 0.09 & 0.09 & 0.08 & -0.01 & -0.02 & -0.06 & -0.33 & -0.38 \\
\hline rank & $1-9$ & $1-9$ & $1-9$ & $1-9$ & $1-9$ & $1-9$ & $1-9$ & $1-9$ & $1-9$ & $10-11$ & $10-11$ & 12 & 13 & 14 \\
\hline
\end{tabular}

Table 10: Head to head comparison for Chinese $\rightarrow$ English systems. 


\begin{tabular}{|c|c|c|c|c|c|c|c|c|c|c|c|c|c|c|}
\hline & 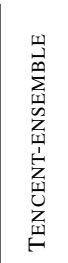 & $\begin{array}{l}\hat{z} \\
\vdots \\
0 \\
0 \\
\vdots \\
b\end{array}$ & 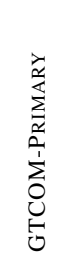 & 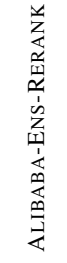 & 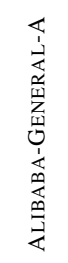 & 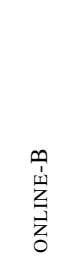 & 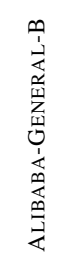 & $\stackrel{\mathrm{s}}{\mathrm{S}}$ & 㫐 & 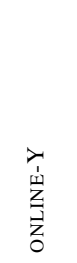 & 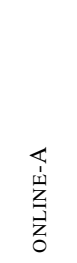 & 艿 & 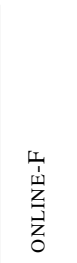 & 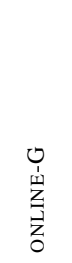 \\
\hline TENCENT-ENSEMBle & - & 0.01 & 0.02 & 0.03 & 0.05 & $0.05 \star$ & 0.05 & $0.13 \ddagger$ & $0.14 \ddagger$ & $0.15 \ddagger$ & $0.18 \ddagger$ & $0.42 \ddagger$ & $0.64 \ddagger$ & $0.65 \ddagger$ \\
\hline UNISOUND & -0.01 & - & 0.01 & 0.02 & 0.03 & 0.04 & 0.04 & $0.11 \ddagger$ & $0.12 \ddagger$ & $0.14 \ddagger$ & $0.17 \ddagger$ & $0.41 \ddagger$ & $0.62 \ddagger$ & $0.64 \ddagger$ \\
\hline GTCOM-PRIMARY & -0.02 & -0.01 & - & 0.01 & 0.03 & $0.03 \star$ & 0.03 & $0.11 \ddagger$ & $0.12 \ddagger$ & $0.13 \ddagger$ & $0.16 \ddagger$ & $0.40 \ddagger$ & $0.62 \ddagger$ & $0.63 \ddagger$ \\
\hline ALIBABA-ENS-RERANK & -0.03 & -0.02 & -0.01 & - & 0.01 & 0.02 & 0.02 & $0.09 \ddagger$ & $0.10 \ddagger$ & $0.12 \ddagger$ & $0.15 \ddagger$ & $0.39 \ddagger$ & $0.60 \ddagger$ & $0.62 \ddagger$ \\
\hline AlibabA-GENERAL-A & -0.05 & -0.03 & -0.03 & -0.01 & - & 0.01 & 0.01 & $0.08 \ddagger$ & $0.09 \dagger$ & $0.10 \ddagger$ & $0.14 \ddagger$ & $0.38 \ddagger$ & $0.59 \ddagger$ & $0.61 \ddagger$ \\
\hline ONLINE-B & -0.05 & -0.04 & -0.03 & -0.02 & -0.01 & - & 0.00 & $0.07 \dagger$ & $0.08 \star$ & $0.10 \ddagger$ & $0.13 \ddagger$ & $0.37 \ddagger$ & $0.58 \ddagger$ & $0.60 \ddagger$ \\
\hline ALIBABA-GENERAL-B & -0.05 & -0.04 & -0.03 & -0.02 & -0.01 & 0.00 & - & $0.07 \dagger$ & $0.08 \dagger$ & $0.10 \ddagger$ & $0.13 \ddagger$ & $0.37 \ddagger$ & $0.58 \ddagger$ & $0.60 \ddagger$ \\
\hline UMD & -0.13 & -0.11 & -0.11 & -0.09 & -0.08 & -0.07 & -0.07 & - & 0.01 & 0.03 & $0.06 \dagger$ & $0.30 \ddagger$ & $0.51 \ddagger$ & $0.53 \ddagger$ \\
\hline NICT & -0.14 & -0.12 & -0.12 & -0.10 & -0.09 & -0.08 & -0.08 & -0.01 & - & 0.01 & $0.04 \dagger$ & $0.28 \ddagger$ & $0.50 \ddagger$ & $0.52 \ddagger$ \\
\hline ONLINE-Y & -0.15 & -0.14 & -0.13 & -0.12 & -0.10 & -0.10 & -0.10 & -0.03 & -0.01 & - & 0.03 & $0.27 \ddagger$ & $0.49 \ddagger$ & $0.50 \ddagger$ \\
\hline ONLINE-A & -0.18 & -0.17 & -0.16 & -0.15 & -0.14 & -0.13 & -0.13 & -0.06 & -0.04 & -0.03 & - & $0.24 \ddagger$ & $0.46 \ddagger$ & $0.47 \ddagger$ \\
\hline UEDIN & -0.42 & -0.41 & -0.40 & -0.39 & -0.38 & -0.37 & -0.37 & -0.30 & -0.28 & -0.27 & -0.24 & - & $0.22 \ddagger$ & $0.23 \ddagger$ \\
\hline ONLINE-F & -0.64 & -0.62 & -0.62 & -0.60 & -0.59 & -0.58 & -0.58 & -0.51 & -0.50 & -0.49 & -0.46 & -0.22 & - & 0.02 \\
\hline ONLINE-G & -0.65 & -0.64 & -0.63 & -0.62 & -0.61 & -0.60 & -0.60 & -0.53 & -0.52 & -0.50 & -0.47 & -0.23 & -0.02 & - \\
\hline & 0.22 & 0.21 & 0.20 & 0.18 & 0.17 & 0.17 & 0.17 & 0.09 & 0.08 & 0.07 & 0.04 & -0.20 & -0.42 & -0.43 \\
\hline rank & $1-7$ & $1-7$ & $1-7$ & $1-7$ & $1-7$ & $1-7$ & $1-7$ & $8-11$ & $8-11$ & $8-11$ & $8-11$ & 12 & $13-14$ & $13-14$ \\
\hline
\end{tabular}

Table 11: Head to head comparison for English $\rightarrow$ Chinese systems.

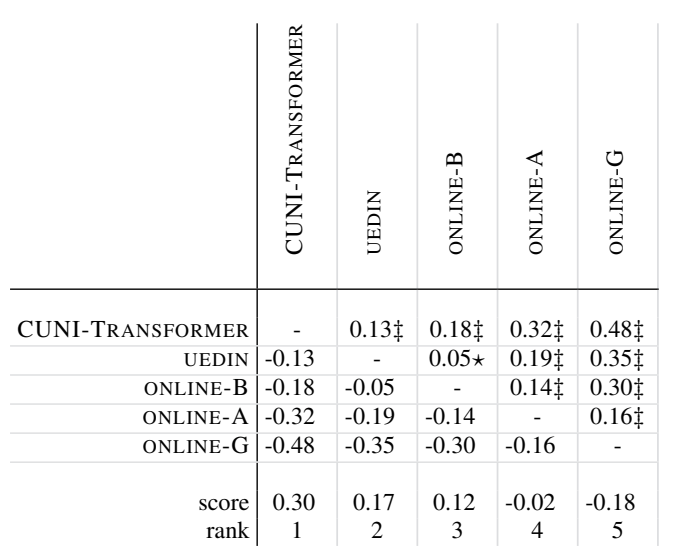

Table 12: Head to head comparison for Czech $\rightarrow$ English systems.

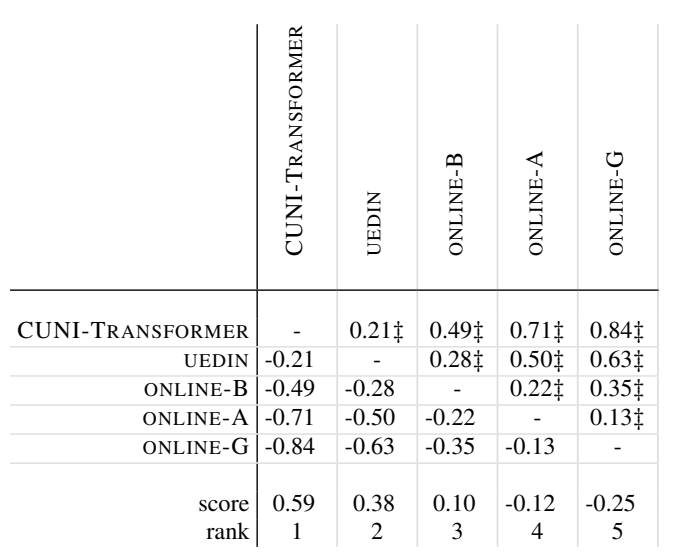

Table 13: Head to head comparison for English $\rightarrow$ Czech systems. 


\begin{tabular}{|c|c|c|c|c|c|c|c|c|c|c|c|c|c|c|c|c|}
\hline & 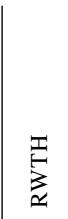 & 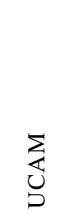 & 卢 & $\begin{array}{l}\frac{n}{1} \\
\text { 崩 } \\
\vec{z} \\
0\end{array}$ & 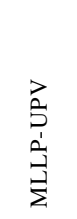 & 主 & 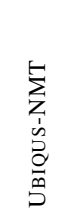 & 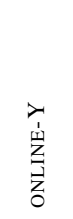 & 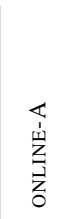 & 古 & $\begin{array}{l}\sum_{n}^{E} \\
z_{1} \\
\sum_{3}\end{array}$ & 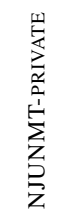 & 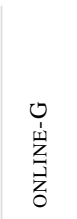 & $\begin{array}{l}\frac{1}{1} \\
\text { 崩 } \\
⿱ 亠 䒑 \\
\\
0\end{array}$ & 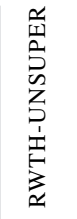 & 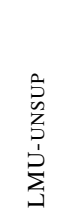 \\
\hline RWTH & - & 0.02 & $0.05 \star$ & $0.07 \ddagger$ & $0.09 \ddagger$ & $0.10 \ddagger$ & $0.10 \ddagger$ & $0.10 \ddagger$ & $0.15 \ddagger$ & $0.15 \ddagger$ & $0.25 \ddagger$ & $0.26 \ddagger$ & $0.49 \ddagger$ & $0.71 \ddagger$ & $1.17 \ddagger$ & $1.25 \ddagger$ \\
\hline UCAM & -0.02 & - & 0.04 & $0.05 \star$ & $0.07 \ddagger$ & $0.08 t$ & $0.08 \pm$ & $0.08 \pm$ & $0.13 \ddagger$ & $0.13 \ddagger$ & $0.23 \ddagger$ & $0.25 \pm$ & $0.47 \pm$ & $0.69 \ddagger$ & $1.15 \ddagger$ & $1.23 \ddagger$ \\
\hline NTT & -0.05 & -0.04 & - & 0.01 & $0.04 \star$ & $0.04 \star$ & $0.04 \dagger$ & $0.05 \dagger$ & $0.09 \ddagger$ & $0.10 \ddagger$ & $0.20 \ddagger$ & $0.21 \ddagger$ & $0.43 \ddagger$ & $0.66 \ddagger$ & $1.11 \ddagger$ & $1.19 \ddagger$ \\
\hline ONLINE-B & -0.07 & -0.05 & -0.01 & - & 0.03 & 0.03 & $0.03 \star$ & 0.04 & $0.08 \ddagger$ & $0.09 \ddagger$ & $0.18 \ddagger$ & $0.20 \ddagger$ & $0.42 \ddagger$ & $0.64 \ddagger$ & $1.10 \ddagger$ & $1.18 \ddagger$ \\
\hline MLLP-UPV & -0.09 & -0.07 & -0.04 & -0.03 & - & 0.00 & 0.01 & 0.01 & $0.05 \dagger$ & $0.06 \star$ & $0.16 \ddagger$ & $0.17 \ddagger$ & $0.40 \ddagger$ & $0.62 \ddagger$ & $1.07 \ddagger$ & $1.16 \ddagger$ \\
\hline JHU & -0.10 & -0.08 & -0.04 & -0.03 & 0.00 & - & 0.00 & 0.01 & $0.05 \dagger$ & $0.06 \dagger$ & $0.15 \ddagger$ & $0.17 \ddagger$ & $0.39 \ddagger$ & $0.61 \ddagger$ & $1.07 \ddagger$ & $1.15 \ddagger$ \\
\hline UBIQUS-NMT & -0.10 & -0.08 & -0.04 & -0.03 & -0.01 & 0.00 & - & 0.01 & $0.05 \star$ & 0.05 & $0.15 \ddagger$ & $0.17 \ddagger$ & $0.39 \ddagger$ & $0.61 \ddagger$ & $1.07 \ddagger$ & $1.15 \ddagger$ \\
\hline ONLINE-Y & -0.10 & -0.08 & -0.05 & -0.04 & -0.01 & -0.01 & -0.01 & - & $0.04 \star$ & $0.05 \star$ & $0.15 \ddagger$ & $0.16 \ddagger$ & $0.38 \ddagger$ & $0.61 \ddagger$ & $1.06 \ddagger$ & $1.15 \ddagger$ \\
\hline ONLINE-A & -0.15 & -0.13 & -0.09 & -0.08 & -0.05 & -0.05 & -0.05 & -0.04 & - & 0.01 & $0.11 \ddagger$ & $0.12 \ddagger$ & $0.34 \ddagger$ & $0.56 \ddagger$ & $1.02 \ddagger$ & $1.10 \ddagger$ \\
\hline UEDIN & -0.15 & -0.13 & -0.10 & -0.09 & -0.06 & -0.06 & -0.05 & -0.05 & -0.01 & - & $0.10 \ddagger$ & $0.11 \ddagger$ & $0.34 \ddagger$ & $0.56 \ddagger$ & $1.01 \ddagger$ & $1.10 \ddagger$ \\
\hline LMU-NMT & -0.25 & -0.23 & -0.20 & -0.18 & -0.16 & -0.15 & -0.15 & -0.15 & -0.11 & -0.10 & - & 0.01 & $0.24 \ddagger$ & $0.46 \ddagger$ & $0.91 \ddagger$ & $1.00 \ddagger$ \\
\hline NJUNMT-PRIVATE & -0.26 & -0.25 & -0.21 & -0.20 & -0.17 & -0.17 & -0.17 & -0.16 & -0.12 & -0.11 & -0.01 & - & $0.22 \ddagger$ & $0.45 \ddagger$ & $0.90 \ddagger$ & $0.98 \ddagger$ \\
\hline ONLINE-G & -0.49 & -0.47 & -0.43 & -0.42 & -0.40 & -0.39 & -0.39 & -0.38 & -0.34 & -0.34 & -0.24 & -0.22 & - & $0.22 \ddagger$ & $0.68 \ddagger$ & $0.76 \ddagger$ \\
\hline ONLINE-F & -0.71 & -0.69 & -0.66 & -0.64 & -0.62 & -0.61 & -0.61 & -0.61 & -0.56 & -0.56 & -0.46 & -0.45 & -0.22 & - & $0.46 \ddagger$ & $0.54 \ddagger$ \\
\hline RWTH-UNSUPER & -1.17 & -1.15 & -1.11 & -1.10 & -1.07 & -1.07 & -1.07 & -1.06 & -1.02 & -1.01 & -0.91 & -0.90 & -0.68 & -0.46 & - & $0.08 \ddagger$ \\
\hline LMU-UNSUP & -1.25 & -1.23 & -1.19 & -1.18 & -1.16 & -1.15 & -1.15 & -1.15 & -1.10 & -1.10 & -1.00 & -0.98 & -0.76 & -0.54 & -0.08 & - \\
\hline score & 0.41 & 0.40 & 0.36 & 0.35 & 0.32 & 0.32 & 0.32 & 0.31 & 0.27 & 0.26 & 0.16 & 0.15 & -0.07 & -0.30 & -0.75 & -0.83 \\
\hline rank & $1-8$ & $1-8$ & $1-8$ & $1-8$ & $1-8$ & $1-8$ & $1-8$ & $1-8$ & $9-10$ & $9-10$ & $11-12$ & $11-12$ & 13 & 14 & 15 & 16 \\
\hline
\end{tabular}

Table 14: Head to head comparison for German $\rightarrow$ English systems.

\begin{tabular}{|c|c|c|c|c|c|c|c|c|c|c|c|c|c|c|c|c|}
\hline & 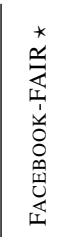 & 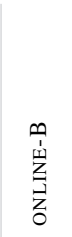 & 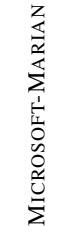 & 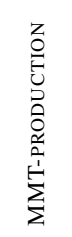 & 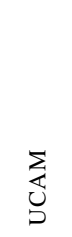 & 点 & $\stackrel{\Xi}{\square}$ & $\begin{array}{l}\searrow \\
\stackrel{1}{\omega} \\
\stackrel{1}{\mid} \\
\vec{z}\end{array}$ & 忌 & $\begin{array}{l}\text { ză } \\
\text { 离 }\end{array}$ & $\begin{array}{l}\sum_{z_{1}}^{5} \\
\sum_{j}\end{array}$ & 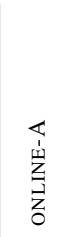 & 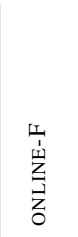 & 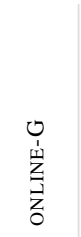 & 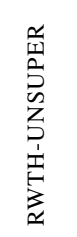 & 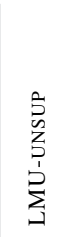 \\
\hline FACEBOOK-FAIR * & - & $0.09 \dagger$ & $0.10 \ddagger$ & $0.11 \ddagger$ & $0.12 \ddagger$ & $0.16 \ddagger$ & $0.20 \ddagger$ & $0.26 \ddagger$ & $0.28 \ddagger$ & $0.30 \ddagger$ & $0.44 \ddagger$ & $0.59 \ddagger$ & $1.04 \ddagger$ & $1.07 \ddagger$ & $1.62 \ddagger$ & $1.77 \ddagger$ \\
\hline ONLINE-B & -0.09 & & 0.01 & 0.02 & 0.02 & $0.07 \dagger$ & $0.11 \ddagger$ & $0.16 \ddagger$ & $0.18 \ddagger$ & $0.21 \ddagger$ & $0.35 \ddagger$ & $0.50 \ddagger$ & $0.95 \ddagger$ & $0.98 \ddagger$ & $1.53 \ddagger$ & $1.68 \ddagger$ \\
\hline MICROSOFT-MARIAN & -0.10 & -0.01 & & 0.01 & 0.01 & $0.06 \star$ & $0.10 \dagger$ & $0.16 \ddagger$ & $0.17 \ddagger$ & $0.20 \ddagger$ & $0.34 \ddagger$ & $0.49 \ddagger$ & $0.94 \ddagger$ & $0.97 \ddagger$ & $1.52 \ddagger$ & $1.67 \ddagger$ \\
\hline MMT-PRODUCTION & -0.11 & -0.02 & -0.01 & - & 0.00 & 0.05 & $0.09 \star$ & $0.14 \ddagger$ & $0.16 \ddagger$ & $0.19 \ddagger$ & $0.33 \ddagger$ & $0.48 \ddagger$ & $0.92 \ddagger$ & $0.95 \ddagger$ & $1.51 \ddagger$ & $1.66 \ddagger$ \\
\hline UCAM & -0.12 & -0.02 & -0.01 & 0.00 & - & 0.05 & $0.08 \star$ & $0.14 \ddagger$ & $0.16 \ddagger$ & $0.19 \ddagger$ & $0.32 \ddagger$ & $0.48 \ddagger$ & $0.92 \ddagger$ & $0.95 \ddagger$ & $1.50 \ddagger$ & $1.66 \pm$ \\
\hline NTT & -0.16 & -0.07 & -0.06 & -0.05 & -0.05 & - & 0.04 & $0.10 \ddagger$ & $0.11 \dagger$ & $0.14 \ddagger$ & $0.28 \ddagger$ & $0.43 \ddagger$ & $0.88 \ddagger$ & $0.91 \ddagger$ & $1.46 \ddagger$ & $1.61 \ddagger$ \\
\hline KIT & -0.20 & -0.11 & -0.10 & -0.09 & -0.08 & -0.04 & - & $0.06 \dagger$ & $0.08 \star$ & $0.10 \dagger$ & $0.24 \ddagger$ & $0.39 \ddagger$ & $0.84 \ddagger$ & $0.87 \ddagger$ & $1.42 \ddagger$ & $1.58 \ddagger$ \\
\hline ONLINE-Y & -0.26 & -0.16 & -0.16 & -0.14 & -0.14 & -0.10 & -0.06 & & 0.02 & 0.04 & $0.18 \ddagger$ & $0.34 \ddagger$ & $0.78 \ddagger$ & $0.81 \ddagger$ & $1.36 \ddagger$ & $1.52 \ddagger$ \\
\hline $\mathrm{JHU}$ & -0.28 & -0.18 & -0.17 & -0.16 & -0.16 & -0.11 & -0.08 & -0.02 & & 0.03 & $0.16 \ddagger$ & $0.32 \ddagger$ & $0.76 \ddagger$ & $0.79 \ddagger$ & $1.34 \ddagger$ & $1.50 \ddagger$ \\
\hline UEDIN & -0.30 & -0.21 & -0.20 & -0.19 & -0.19 & -0.14 & -0.10 & -0.04 & -0.03 & - & $0.14 \ddagger$ & $0.29 \ddagger$ & $0.74 \ddagger$ & $0.77 \ddagger$ & $1.32 \ddagger$ & $1.47 \ddagger$ \\
\hline LMU-NMT & -0.44 & -0.35 & -0.34 & -0.33 & -0.32 & -0.28 & -0.24 & -0.18 & -0.16 & -0.14 & - & $0.15 \ddagger$ & $0.60 \ddagger$ & $0.63 \ddagger$ & $1.18 \ddagger$ & $1.33 \ddagger$ \\
\hline ONLINE-A & -0.59 & -0.50 & -0.49 & -0.48 & -0.48 & -0.43 & -0.39 & -0.34 & -0.32 & -0.29 & -0.15 & - & $0.44 \ddagger$ & $0.48 \ddagger$ & $1.03 \ddagger$ & $1.18 \ddagger$ \\
\hline ONLINE-F & -1.04 & -0.95 & -0.94 & -0.92 & -0.92 & -0.88 & -0.84 & -0.78 & -0.76 & -0.74 & -0.60 & -0.44 & & 0.03 & $0.58 \ddagger$ & $0.74 \ddagger$ \\
\hline ONLINE-G & -1.07 & -0.98 & -0.97 & -0.95 & -0.95 & -0.91 & -0.87 & -0.81 & -0.79 & -0.77 & -0.63 & -0.48 & -0.03 & - & $0.55 \ddagger$ & $0.71 \ddagger$ \\
\hline RWTH-UNSUPER & -1.62 & -1.53 & -1.52 & -1.51 & -1.50 & -1.46 & -1.42 & -1.36 & -1.34 & -1.32 & -1.18 & -1.03 & -0.58 & -0.55 & - & $0.16 \ddagger$ \\
\hline LMU-UNSUP & -1.77 & -1.68 & -1.67 & -1.66 & -1.66 & -1.61 & -1.58 & -1.52 & -1.50 & -1.47 & -1.33 & -1.18 & -0.74 & -0.71 & -0.16 & - \\
\hline & 0.65 & 0.56 & 0.55 & 0.54 & 0.54 & 0.49 & 0.45 & 0.40 & 0.38 & 0.35 & 0.21 & 0.06 & -0.39 & -0.42 & -0.97 & -1.12 \\
\hline rank & 1 & $2-7$ & $2-7$ & $2-7$ & $2-7$ & $2-7$ & $2-7$ & $8-10$ & $8-10$ & $8-10$ & 11 & 12 & $13-14$ & $13-14$ & 15 & 16 \\
\hline
\end{tabular}

Table 15: Head to head comparison for English $\rightarrow$ German systems. 


\begin{tabular}{|c|c|c|c|c|c|c|c|c|c|c|c|c|c|c|}
\hline & 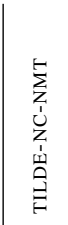 & $\stackrel{\text { 㫐 }}{\text { 乙 }}$ & 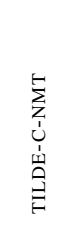 & 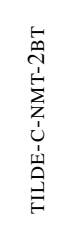 & 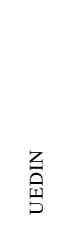 & 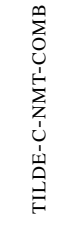 & $\begin{array}{l}\infty \\
1 \\
\omega \\
\underline{\omega} \\
\vec{z} \\
0\end{array}$ & 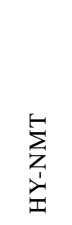 & $\begin{array}{l}U \\
0 \\
\vdots \\
\vdots \\
\vdots \\
\vdots \\
0\end{array}$ & 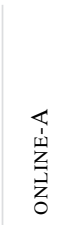 & 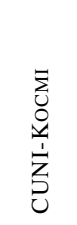 & 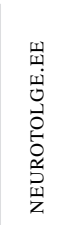 & $\begin{array}{l}0 \\
1 \\
\text { 岁 } \\
\underline{z} \\
\vdots \\
0\end{array}$ & 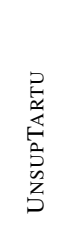 \\
\hline TILDE-NC-NMT & - & $0.09 \ddagger$ & $0.11 \ddagger$ & $0.14 \ddagger$ & $0.14 \ddagger$ & $0.16 \ddagger$ & $0.21 \ddagger$ & $0.22 \ddagger$ & $0.22 \ddagger$ & $0.26 \ddagger$ & $0.32 \ddagger$ & $0.44 \ddagger$ & $0.67 \ddagger$ & $1.28 \ddagger$ \\
\hline NICT & -0.09 & - & 0.02 & $0.05 \star$ & 0.05 & $0.07 \dagger$ & $0.12 \ddagger$ & $0.13 \ddagger$ & $0.13 \ddagger$ & $0.18 \ddagger$ & $0.23 \ddagger$ & $0.36 \ddagger$ & $0.58 \ddagger$ & $1.19 \ddagger$ \\
\hline TILDE-C-NMT & -0.11 & -0.02 & - & 0.03 & 0.03 & 0.04 & $0.10 \ddagger$ & $0.11 \dagger$ & $0.11 \ddagger$ & $0.15 \ddagger$ & $0.21 \ddagger$ & $0.33 \ddagger$ & $0.56 \ddagger$ & $1.17 \ddagger$ \\
\hline TILDE-C-NMT-2BT & -0.14 & -0.05 & -0.03 & - & 0.00 & 0.02 & $0.07 \dagger$ & $0.08 \dagger$ & $0.08 \dagger$ & $0.12 \ddagger$ & $0.18 \ddagger$ & $0.30 \ddagger$ & $0.53 \ddagger$ & $1.14 \ddagger$ \\
\hline UEDIN & -0.14 & -0.05 & -0.03 & 0.00 & - & 0.02 & $0.07 \dagger$ & $0.08 \dagger$ & $0.08 \dagger$ & $0.12 \ddagger$ & $0.18 \ddagger$ & $0.30 \ddagger$ & $0.53 \ddagger$ & $1.14 \ddagger$ \\
\hline TILDE-C-NMT-COMB & -0.16 & -0.07 & -0.04 & -0.02 & -0.02 & - & $0.05 \star$ & 0.06 & $0.06 \star$ & $0.11 \ddagger$ & $0.16 \ddagger$ & $0.29 \ddagger$ & $0.51 \ddagger$ & $1.12 \ddagger$ \\
\hline ONLINE-B & -0.21 & -0.12 & -0.10 & -0.07 & -0.07 & -0.05 & - & 0.01 & 0.01 & $0.05 \star$ & $0.11 \dagger$ & $0.23 \ddagger$ & $0.46 \ddagger$ & $1.07 \ddagger$ \\
\hline HY-NMT & -0.22 & -0.13 & -0.11 & -0.08 & -0.08 & -0.06 & -0.01 & - & 0.00 & $0.04 \star$ & $0.10 \dagger$ & $0.22 \ddagger$ & $0.45 \ddagger$ & $1.06 \ddagger$ \\
\hline TALP-UPC & -0.22 & -0.13 & -0.11 & -0.08 & -0.08 & -0.06 & -0.01 & 0.00 & - & $0.04 \star$ & $0.10 \dagger$ & $0.22 \ddagger$ & $0.45 \ddagger$ & $1.06 \ddagger$ \\
\hline ONLINE-A & $\mid-0.26$ & -0.18 & -0.15 & -0.12 & -0.12 & -0.11 & -0.05 & -0.04 & -0.04 & - & 0.06 & $0.18 \ddagger$ & $0.40 \ddagger$ & $1.01 \ddagger$ \\
\hline CUNI-КосмI & -0.32 & -0.23 & -0.21 & -0.18 & -0.18 & -0.16 & -0.11 & -0.10 & -0.10 & -0.06 & - & $0.12 \ddagger$ & $0.35 \ddagger$ & $0.96 \ddagger$ \\
\hline NEUROTOLGE.EE & $\mid-0.44$ & -0.36 & -0.33 & -0.30 & -0.30 & -0.29 & -0.23 & -0.22 & -0.22 & -0.18 & -0.12 & - & $0.22 \ddagger$ & $0.83 \ddagger$ \\
\hline ONLINE-G & -0.67 & -0.58 & -0.56 & -0.53 & -0.53 & -0.51 & -0.46 & -0.45 & -0.45 & -0.40 & -0.35 & -0.22 & - & $0.61 \ddagger$ \\
\hline UNSUPTARTU & -1.28 & -1.19 & -1.17 & -1.14 & -1.14 & -1.12 & -1.07 & -1.06 & -1.06 & -1.01 & -0.96 & -0.83 & -0.61 & - \\
\hline & 0.33 & 0.24 & 0.21 & 0.19 & 0.19 & 0.17 & 0.12 & 0.11 & 0.11 & 0.06 & 0.01 & -0.12 & -0.34 & -0.95 \\
\hline & 1 & $2-9$ & $2-9$ & $2-9$ & $2-9$ & $2-9$ & $2-9$ & $2-9$ & $2-9$ & $10-11$ & $10-11$ & 12 & 13 & 14 \\
\hline
\end{tabular}

Table 16: Head to head comparison for Estonian $\rightarrow$ English systems.

\begin{tabular}{|c|c|c|c|c|c|c|c|c|c|c|c|c|c|c|}
\hline & 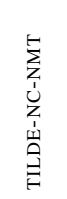 & $\underset{乙}{己}$ & 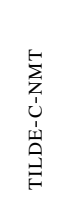 & 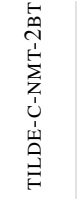 & 晃 & 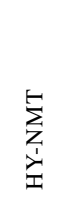 & $\begin{array}{l}\text { za } \\
\text { 凷 }\end{array}$ & 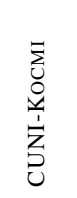 & 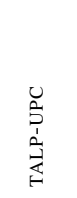 & 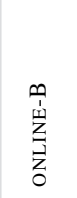 & 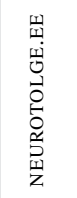 & 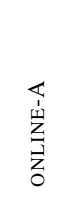 & 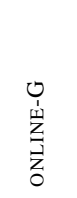 & 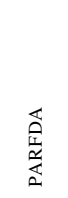 \\
\hline TILDE-NC-NMT & - & $0.10 \star$ & $0.12 \dagger$ & $0.13 \ddagger$ & $0.21 \ddagger$ & $0.22 \ddagger$ & $0.25 \ddagger$ & $0.33 \ddagger$ & $0.37 \ddagger$ & $0.45 \ddagger$ & $0.68 \ddagger$ & $0.74 \ddagger$ & $0.95 \ddagger$ & $1.07 \ddagger$ \\
\hline NICT & -0.10 & - & 0.03 & 0.03 & $0.11 \dagger$ & $0.12 \dagger$ & $0.16 \ddagger$ & $0.24 \ddagger$ & $0.27 \ddagger$ & $0.36 \ddagger$ & $0.58 \ddagger$ & $0.65 \ddagger$ & $0.86 \ddagger$ & $0.97 \ddagger$ \\
\hline TILDE-C-NMT & -0.12 & -0.03 & - & 0.01 & $0.09 \star$ & $0.10 \star$ & $0.13 \dagger$ & $0.21 \ddagger$ & $0.25 \ddagger$ & $0.33 \ddagger$ & $0.56 \ddagger$ & $0.62 \ddagger$ & $0.83 \ddagger$ & $0.95 \ddagger$ \\
\hline TILDE-C-NMT-2BT & -0.13 & -0.03 & -0.01 & - & $0.08 \star$ & $0.09 \star$ & $0.12 \dagger$ & $0.20 \ddagger$ & $0.24 \ddagger$ & $0.32 \ddagger$ & $0.55 \ddagger$ & $0.61 \ddagger$ & $0.82 \ddagger$ & $0.94 \ddagger$ \\
\hline AALTO & -0.21 & -0.11 & -0.09 & -0.08 & - & 0.01 & 0.05 & $0.12 \dagger$ & $0.16 \ddagger$ & $0.24 \ddagger$ & $0.47 \ddagger$ & $0.54 \ddagger$ & $0.75 \ddagger$ & $0.86 \ddagger$ \\
\hline HY-NMT & -0.22 & -0.12 & -0.10 & -0.09 & -0.01 & - & 0.03 & $0.11 \dagger$ & $0.15 \ddagger$ & $0.23 \ddagger$ & $0.46 \ddagger$ & $0.52 \ddagger$ & $0.73 \ddagger$ & $0.85 \ddagger$ \\
\hline UEDIN & -0.25 & -0.16 & -0.13 & -0.12 & -0.05 & -0.03 & - & $0.08 \star$ & $0.11 \dagger$ & $0.20 \ddagger$ & $0.43 \ddagger$ & $0.49 \ddagger$ & $0.70 \ddagger$ & $0.81 \ddagger$ \\
\hline CUNI-KосMI & -0.33 & -0.24 & -0.21 & -0.20 & -0.12 & -0.11 & -0.08 & - & 0.04 & $0.12 \dagger$ & $0.35 \ddagger$ & $0.41 \ddagger$ & $0.62 \ddagger$ & $0.74 \ddagger$ \\
\hline TALP-UPC & -0.37 & -0.27 & -0.25 & -0.24 & -0.16 & -0.15 & -0.11 & -0.04 & - & $0.08 \star$ & $0.31 \ddagger$ & $0.38 \ddagger$ & $0.59 \ddagger$ & $0.70 \ddagger$ \\
\hline ONLINE-B & -0.45 & -0.36 & -0.33 & -0.32 & -0.24 & -0.23 & -0.20 & -0.12 & -0.08 & - & $0.23 \ddagger$ & $0.29 \ddagger$ & $0.50 \ddagger$ & $0.62 \ddagger$ \\
\hline NEUROTOLGE.EE & -0.68 & -0.58 & -0.56 & -0.55 & -0.47 & -0.46 & -0.43 & -0.35 & -0.31 & -0.23 & - & $0.06 \star$ & $0.27 \ddagger$ & $0.39 \ddagger$ \\
\hline ONLINE-A & -0.74 & -0.65 & -0.62 & -0.61 & -0.54 & -0.52 & -0.49 & -0.41 & -0.38 & -0.29 & -0.06 & - & $0.21 \ddagger$ & $0.32 \ddagger$ \\
\hline ONLINE-G & -0.95 & -0.86 & -0.83 & -0.82 & -0.75 & -0.73 & -0.70 & -0.62 & -0.59 & -0.50 & -0.27 & -0.21 & - & $0.11 \ddagger$ \\
\hline PARFDA & -1.07 & -0.97 & -0.95 & -0.94 & -0.86 & -0.85 & -0.81 & -0.74 & -0.70 & -0.62 & -0.39 & -0.32 & -0.11 & - \\
\hline score & 0.55 & 0.45 & 0.43 & 0.42 & 0.34 & 0.33 & 0.29 & 0.22 & 0.18 & 0.10 & -0.13 & -0.20 & -0.41 & -0.52 \\
\hline rank & 1 & $2-4$ & $2-4$ & $2-4$ & $5-7$ & $5-7$ & $5-7$ & $8-9$ & $8-9$ & 10 & 11 & 12 & 13 & 14 \\
\hline
\end{tabular}

Table 17: Head to head comparison for English $\rightarrow$ Estonian systems.

\begin{tabular}{|c|c|c|c|c|c|c|c|c|c|}
\hline & $\stackrel{\sqcup}{己}$ & 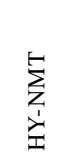 & $\begin{array}{l}\text { z } \\
\text { 站 }\end{array}$ & 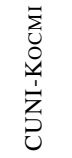 & 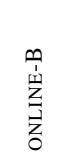 & 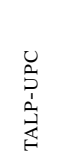 & 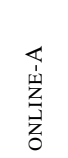 & 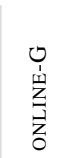 & $\begin{array}{l}\sum_{Z}^{E} \\
\text { 足 } \\
\text { D }\end{array}$ \\
\hline NICT & - & 0.02 & 0.05 & $0.07 \dagger$ & $0.07 \dagger$ & $0.11 \ddagger$ & $0.11 \ddagger$ & $0.29 \ddagger$ & $0.56 \ddagger$ \\
\hline HY-NMT & -0.02 & - & 0.03 & 0.05 ᄎ & $0.05 \star$ & $0.08 \dagger$ & $0.08 \dagger$ & $0.26 \mp$ & $0.53 \ddagger$ \\
\hline UEDIN & -0.05 & -0.03 & - & 0.02 & 0.02 & $0.06 \dagger$ & $0.06 \dagger$ & $0.24 \ddagger$ & $0.51 \ddagger$ \\
\hline CUNI-KосMI & -0.07 & -0.05 & -0.02 & - & 0.00 & 0.04 & 0.04 & $0.22 \ddagger$ & $0.49 \ddagger$ \\
\hline ONLINE-B & -0.07 & -0.05 & -0.02 & 0.00 & - & 0.03 & 0.03 & $0.21 \ddagger$ & $0.48 \ddagger$ \\
\hline TALP-UPC & -0.11 & -0.08 & -0.06 & -0.04 & -0.03 & - & 0.00 & $0.18 \pm$ & $0.45 \ddagger$ \\
\hline ONLINE-A & -0.11 & -0.08 & -0.06 & -0.04 & -0.03 & 0.00 & - & $0.18 \ddagger$ & $0.45 \ddagger$ \\
\hline ONLINE-G & -0.29 & -0.26 & -0.24 & -0.22 & -0.21 & -0.18 & -0.18 & - & $0.27 \ddagger$ \\
\hline JUCBNMT & -0.56 & -0.53 & -0.51 & -0.49 & -0.48 & -0.45 & -0.45 & -0.27 & - \\
\hline & 0.15 & 0.13 & 0.10 & 0.08 & 0.08 & 0.05 & 0.04 & -0.13 & -0.40 \\
\hline anl & $1-7$ & $1-7$ & $1-7$ & $1-7$ & $1-7$ & $1-7$ & $1-7$ & 8 & 9 \\
\hline
\end{tabular}

Table 18: Head to head comparison for Finnish $\rightarrow$ English systems. 


\begin{tabular}{|c|c|c|c|c|c|c|c|c|c|c|c|c|}
\hline & $\stackrel{\text { 㫐 }}{\text { 乙 }}$ & 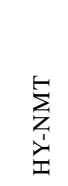 & 쫌 & 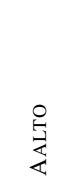 & 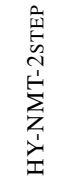 & 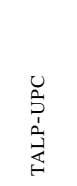 & 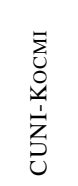 & 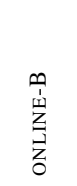 & 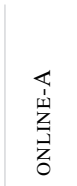 & $\begin{array}{l}0 \\
\mathbf{1} \\
\text { 岌 } \\
⿱ 亠 䒑 \\
\mathbf{Z}\end{array}$ & $\begin{array}{l}\sum_{\infty}^{E} \\
\stackrel{1}{ \pm} \\
i\end{array}$ & 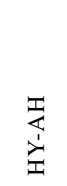 \\
\hline NICT & - & 0.05 & $0.20 \ddagger$ & $0.25 \ddagger$ & $0.26 \ddagger$ & $0.28 \ddagger$ & $0.34 \ddagger$ & $0.34 \ddagger$ & $0.73 \ddagger$ & $0.75 \ddagger$ & $0.86 \ddagger$ & $0.89 \ddagger$ \\
\hline HY-NMT & -0.05 & - & $0.14 \dagger$ & $0.19 \ddagger$ & $0.21 \ddagger$ & $0.23 \ddagger$ & $0.28 \ddagger$ & $0.28 \ddagger$ & $0.68 \ddagger$ & $0.70 \ddagger$ & $0.80 \ddagger$ & $0.83 \ddagger$ \\
\hline UEDIN & -0.20 & -0.14 & - & 0.05 & $0.07^{\circ}$ & $0.09 \star$ & $0.14 \dagger$ & $0.14 \ddagger$ & $0.54 \ddagger$ & $0.56 \ddagger$ & $0.66 \ddagger$ & $0.69 \ddagger$ \\
\hline AALTo & -0.25 & -0.19 & -0.05 & - & 0.01 & 0.03 & $0.09 \star$ & $0.09 \star$ & $0.48 \ddagger$ & $0.50 \ddagger$ & $0.61 \ddagger$ & $0.64 \ddagger$ \\
\hline HY-NMT-2STEP & -0.26 & -0.21 & -0.07 & -0.01 & - & 0.02 & 0.07 & $0.07 \star$ & $0.47 \ddagger$ & $0.49 \ddagger$ & $0.59 \ddagger$ & $0.63 \ddagger$ \\
\hline TALP-UPC & -0.28 & -0.23 & -0.09 & -0.03 & -0.02 & - & 0.05 & 0.05 & $0.45 \ddagger$ & $0.47 \ddagger$ & $0.57 \ddagger$ & $0.61 \ddagger$ \\
\hline CUNI-KосмI & -0.34 & -0.28 & -0.14 & -0.09 & -0.07 & -0.05 & - & 0.00 & $0.40 \ddagger$ & $0.42 \ddagger$ & $0.52 \ddagger$ & $0.55 \ddagger$ \\
\hline ONLINE-B & -0.34 & -0.28 & -0.14 & -0.09 & -0.07 & -0.05 & 0.00 & - & $0.39 \ddagger$ & $0.42 \ddagger$ & $0.52 \ddagger$ & $0.55 \ddagger$ \\
\hline ONLINE-A & -0.73 & -0.68 & -0.54 & -0.48 & -0.47 & -0.45 & -0.40 & -0.39 & - & 0.02 & $0.12 \dagger$ & $0.16 \ddagger$ \\
\hline ONLINE-G & -0.75 & -0.70 & -0.56 & -0.50 & -0.49 & -0.47 & -0.42 & -0.42 & -0.02 & - & $0.10 \dagger$ & $0.14 \ddagger$ \\
\hline HY-SMT & -0.86 & -0.80 & -0.66 & -0.61 & -0.59 & -0.57 & -0.52 & -0.52 & -0.12 & -0.10 & - & 0.03 \\
\hline HY-AH & -0.89 & -0.83 & -0.69 & -0.64 & -0.63 & -0.61 & -0.55 & -0.55 & -0.16 & -0.14 & -0.03 & - \\
\hline score & 0.52 & 0.47 & 0.32 & 0.27 & 0.26 & 0.24 & 0.18 & 0.18 & -0.21 & -0.23 & -0.33 & -0.37 \\
\hline Taink & $1-2$ & $1-2$ & $3-8$ & $3-8$ & $3-8$ & $3-8$ & $3-8$ & $3-8$ & $9-10$ & $9-10$ & $11-12$ & $11-12$ \\
\hline
\end{tabular}

Table 19: Head to head comparison for English $\rightarrow$ Finnish systems.

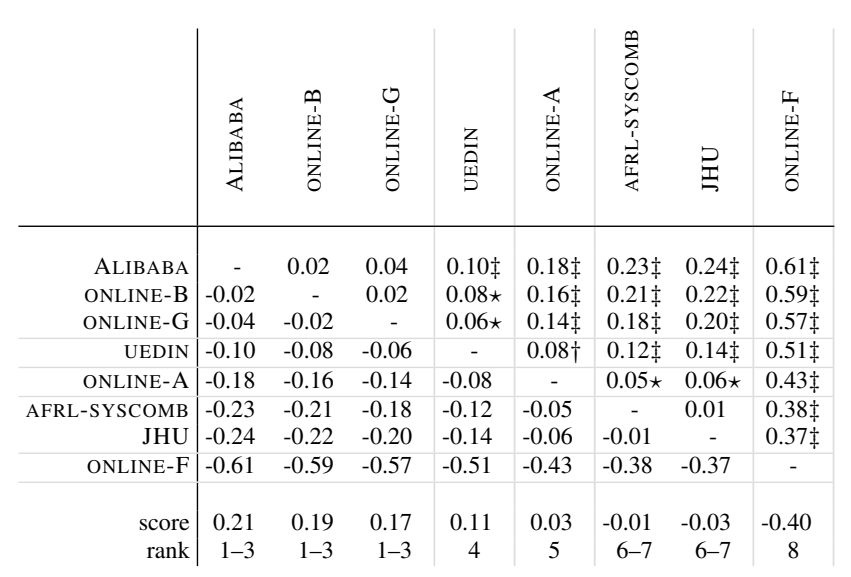

Table 20: Head to head comparison for Russian $\rightarrow$ English systems.

\begin{tabular}{|c|c|c|c|c|c|c|c|c|c|}
\hline & 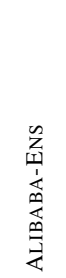 & $\begin{array}{l}0 \\
\text { U } \\
\text { 湈 } \\
\vec{Z}\end{array}$ & 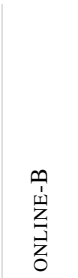 & $\begin{array}{l}\text { Z } \\
\text { 兽 }\end{array}$ & 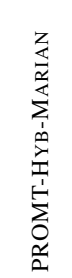 & 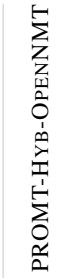 & 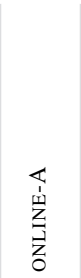 & 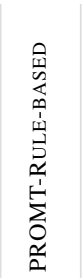 & $\begin{array}{l}\frac{1}{1} \\
\frac{1}{z} \\
\vec{J} \\
\end{array}$ \\
\hline ALIBABA-ENS & & 0.03 & $0.19 \ddagger$ & $0.21 \ddagger$ & $0.24 \ddagger$ & $0.29 \ddagger$ & $0.36 \ddagger$ & $0.43 \ddagger$ & $0.93 \ddagger$ \\
\hline ONLINE-G & -0.03 & - & $0.16 \ddagger$ & $0.18 \ddagger$ & $0.21 \ddagger$ & $0.26 \ddagger$ & $0.33 \ddagger$ & $0.40 \ddagger$ & $0.90 \ddagger$ \\
\hline ONLINE-B & -0.19 & -0.16 & & 0.01 & $0.04 \star$ & $0.09 \ddagger$ & $0.16 \ddagger$ & $0.23 \ddagger$ & $0.74 \ddagger$ \\
\hline UEDIN & -0.21 & -0.18 & -0.01 & - & 0.03 & $0.08 \dagger$ & $0.15 \ddagger$ & $0.22 \pm$ & $0.72 \ddagger$ \\
\hline PROMT-HYB-MARIAN & -0.24 & -0.21 & -0.04 & -0.03 & & 0.05 ᄎ & $0.12 \ddagger$ & $0.19 \ddagger$ & $0.69 \ddagger$ \\
\hline PROMT-HYB-OPENNMT & -0.29 & -0.26 & -0.09 & -0.08 & -0.05 & & $0.07 \dagger$ & $0.14 \ddagger$ & $0.65 \ddagger$ \\
\hline ONLINE-A & -0.36 & -0.33 & -0.16 & -0.15 & -0.12 & -0.07 & & $0.07 \dagger$ & $0.58 \ddagger$ \\
\hline PROMT-RULE-BASED & -0.43 & -0.40 & -0.23 & -0.22 & -0.19 & -0.14 & -0.07 & - & $0.50 \ddagger$ \\
\hline ONLINE-F & -0.93 & -0.90 & -0.74 & -0.72 & -0.69 & -0.65 & -0.58 & -0.50 & - \\
\hline $\begin{array}{r}\text { score } \\
\text { rank }\end{array}$ & $\begin{array}{r}0.35 \\
1-2\end{array}$ & $\begin{array}{r}0.32 \\
1-2\end{array}$ & $\begin{array}{r}0.16 \\
3-5\end{array}$ & $\begin{array}{r}0.14 \\
3-5\end{array}$ & $\begin{array}{r}0.12 \\
3-5\end{array}$ & $\begin{array}{c}0.07 \\
6\end{array}$ & $\begin{array}{c}-0.00 \\
7\end{array}$ & $\begin{array}{c}-0.07 \\
8\end{array}$ & $\begin{array}{c}-0.58 \\
9\end{array}$ \\
\hline
\end{tabular}

Table 21: Head to head comparison for English $\rightarrow$ Russian systems. 


\begin{tabular}{|c|c|c|c|c|c|c|}
\hline & 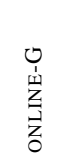 & 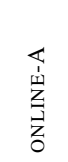 & 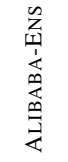 & 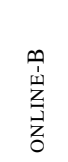 & 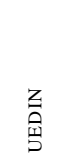 & 它 \\
\hline ONLINE-G & - & 0.02 & $0.06 \star$ & $0.06 \dagger$ & $0.11 \ddagger$ & $0.13 \ddagger$ \\
\hline ONLINE-A & -0.02 & - & 0.04 & 0.04 & $0.08 \star$ & $0.10 \ddagger$ \\
\hline ALIBABA-ENS & -0.06 & -0.04 & - & 0.01 & 0.05 & $0.07 \dagger$ \\
\hline ONLINE-B & -0.06 & -0.04 & -0.01 & - & 0.04 & $0.06 \star$ \\
\hline UEDIN & -0.11 & -0.08 & -0.05 & -0.04 & - & 0.02 \\
\hline NICT & -0.13 & -0.10 & -0.07 & -0.06 & -0.02 & - \\
\hline score & 0.09 & 0.07 & 0.03 & 0.02 & -0.02 & -0.04 \\
\hline ran & $1-6$ & $1-6$ & $1-6$ & $1-6$ & $1-6$ & $1-6$ \\
\hline
\end{tabular}

Table 22: Head to head comparison for Turkish $\rightarrow$ English systems.

\begin{tabular}{|c|c|c|c|c|c|c|c|c|}
\hline & 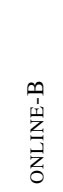 & $\begin{array}{l}\text { 금 } \\
\text { 思 }\end{array}$ & 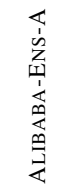 & 它 & 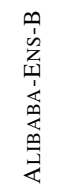 & 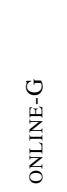 & $\underset{⿱ 乛}{\stackrel{I}{\mid}}$ & 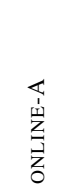 \\
\hline ONLINE-B & - & 0.05 & 0.06 & $0.15 \star$ & $0.17 \dagger$ & $0.22 \ddagger$ & $0.34 \ddagger$ & $0.53 \ddagger$ \\
\hline UEDIN & -0.05 & - & 0.01 & $0.09 \star$ & $0.11 \star$ & $0.16 \dagger$ & $0.28 \ddagger$ & $0.48 \ddagger$ \\
\hline ALIBABA-ENS-A & -0.06 & -0.01 & - & 0.09 & 0.10 & $0.16 \dagger$ & $0.28 \ddagger$ & $0.47 \ddagger$ \\
\hline NICT & -0.15 & -0.09 & -0.09 & - & 0.02 & 0.07 & $0.19 \ddagger$ & $0.38 \ddagger$ \\
\hline ALIBABA-ENS-B & -0.17 & -0.11 & -0.10 & -0.02 & - & 0.05 & $0.17 \dagger$ & $0.36 \ddagger$ \\
\hline ONLINE-G & -0.22 & -0.16 & -0.16 & -0.07 & -0.05 & - & $0.12 \star$ & $0.31 \ddagger$ \\
\hline RWTH & -0.34 & -0.28 & -0.28 & -0.19 & -0.17 & -0.12 & - & $0.19 \ddagger$ \\
\hline ONLINE-A & -0.53 & -0.48 & -0.47 & -0.38 & -0.36 & -0.31 & -0.19 & - \\
\hline score & 0.28 & 0.22 & 0.22 & 0.13 & 0.11 & 0.06 & -0.06 & -0.25 \\
\hline rank & $1-6$ & $1-6$ & $1-6$ & $1-6$ & $1-6$ & $1-6$ & 7 & 8 \\
\hline
\end{tabular}

Table 23: Head to head comparison for English $\rightarrow$ Turkish systems.

\begin{tabular}{|c|c|c|c|c|c|c|}
\hline & 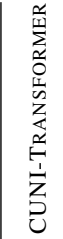 & $\begin{array}{l}\text { ż } \\
\text { 凷 }\end{array}$ & 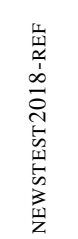 & 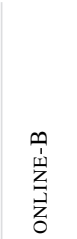 & 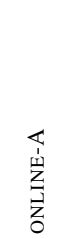 & 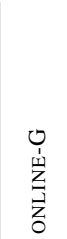 \\
\hline CUNI-TRANSFORMER & - & $0.15 \ddagger$ & $0.18 \ddagger$ & $0.54 \ddagger$ & $0.85 \ddagger$ & $1.02 \ddagger$ \\
\hline UEDIN & -0.15 & - & 0.04 & $0.39 \ddagger$ & $0.70 \ddagger$ & $0.88 \pm$ \\
\hline NEWSTEST2018-REF & -0.18 & -0.04 & - & $0.36 \ddagger$ & $0.66 \ddagger$ & $0.84 \ddagger$ \\
\hline ONLINE-B & -0.54 & -0.39 & -0.36 & - & $0.31 \ddagger$ & $0.48 \ddagger$ \\
\hline ONLINE-A & -0.85 & -0.70 & -0.66 & -0.31 & - & $0.18 \ddagger$ \\
\hline ONLINE-G & -1.02 & -0.88 & -0.84 & -0.48 & -0.18 & - \\
\hline score & 0.67 & 0.52 & 0.48 & 0.13 & -0.18 & -0.35 \\
\hline rank & 1 & $2-3$ & $2-3$ & 4 & 5 & 6 \\
\hline
\end{tabular}

Table 24: Head to head comparison for Czech $\rightarrow$ English systems, based on source-based DA. 